\title{
Article \\ Families of Solutions of Multitemporal Nonlinear Schrödinger PDE
}

\author{
Cristian Ghiu $^{1,+(\mathbb{D}) \text {, Constantin Udriste }}{ }^{2, *,+, \pm(\mathbb{D})}$ and Lavinia Laura Petrescu ${ }^{2,+}$ \\ 1 Department of Mathematical Methods and Models, Faculty of Applied Sciences, University Politehnica of \\ Bucharest, Splaiul Independentei 313, Sector 6, RO-060042 Bucharest, Romania; crisghiu@yahoo.com \\ 2 Department of Mathematics and Informatics, Faculty of Applied Sciences, University Politehnica of Bucharest, \\ Splaiul Independentei 313, Sector 6, RO-060042 Bucharest, Romania; lavinialaurapetrescu@yahoo.com \\ * Correspondence: udriste@mathem.pub.ro; Tel.: +40-745-371-684 \\ + These authors contributed equally to this work. \\ ‡ Second address: Academy of Romanian Scientists, Ilfov 3, Sector 5, RO-050044 Bucharest, Romania.
}

check for updates

Citation: Ghiu, C.; Udriste, C.;

Petrescu, L.L. Families of Solutions of Multitemporal Nonlinear

Schrödinger PDE. Mathematics 2021, 9 , 1995. https://doi.org/10.3390/ math9161995

Academic Editors: Alberto Ferrero and Luigi Rodino

Received: 30 June 2021

Accepted: 16 August 2021

Published: 20 August 2021

Publisher's Note: MDPI stays neutral with regard to jurisdictional claims in published maps and institutional affiliations.

Copyright: (C) 2021 by the authors. Licensee MDPI, Basel, Switzerland. This article is an open access article distributed under the terms and conditions of the Creative Commons Attribution (CC BY) license (https:/ / creativecommons.org/licenses/by/ $4.0 /)$.
Abstract: The multitemporal nonlinear Schrödinger PDE (with oblique derivative) was stated for the first time in our research group as a universal amplitude equation which can be derived via a multiple scaling analysis in order to describe slow modulations of the envelope of a spatially and temporarily oscillating wave packet in space and multitime (an equation which governs the dynamics of solitons through meta-materials). Now we exploit some hypotheses in order to find important explicit families of exact solutions in all dimensions for the multitime nonlinear Schrödinger PDE with a multitemporal directional derivative term. Using quite effective methods, we discovered families of ODEs and PDEs whose solutions generate solutions of multitime nonlinear Schrödinger PDE. Each new construction involves a relatively small amount of intermediate calculations.

Keywords: multitime nonlinear Schrödinger PDE; oblique derivative; exact solutions; first integrals

MSC: 35Q41; 35Q55

\section{Introduction}

In order to define the multitime nonlinear Schrödinger PDE (multitime NLSE), we need: (i) Two intervals, $I_{1} \subseteq \mathbb{R}, I_{2} \subseteq\left[0, \infty\right.$ ), (ii) an open subset $D \subseteq \mathbb{R}^{m}$, (iii) the function $f: I_{1} \times I_{2} \rightarrow \mathbb{R}$, and (iv) the vector field $h=\left(h^{\alpha}\right): I_{1} \times D \rightarrow \mathbb{R}^{m}$ (for $\alpha \in\{1,2, \ldots, m\}$ ). All these together define the multitime NLSE [1]

$$
i h^{\alpha}(x, t) \frac{\partial u}{\partial t^{\alpha}}+\frac{\partial^{2} u}{\partial x^{2}}+f\left(x,|u|^{2}\right) u=0
$$

Remark 1. (a) If $u$ is a solution of multitime NLSE (1), then also - $u$ is a solution of the multitime NLSE (1).

(b) If $u$ is a solution of the multitime NLSE (1), then for any constant $k \in \mathbb{R}$, the function $\varphi:=e^{i k} u$ is a solution of the multitime NLSE (1).

(c) If $u$ is a solution of the multitime NLSE (1), then for any pair of constants $\rho, k \in \mathbb{R}, \rho \neq 0$, the function $\varphi:=\rho e^{i k} u$ is a solution of the multitime NLSE

$$
i h^{\alpha}(x, t) \frac{\partial \varphi}{\partial t^{\alpha}}+\frac{\partial^{2} \varphi}{\partial x^{2}}+f\left(x, \frac{1}{\rho^{2}}|\varphi|^{2}\right) \varphi=0 .
$$

(d) If we introduce the Hamiltonian action $H_{t}(u)=\frac{1}{2} \int\left(\left|u_{x}\right|^{2}-g\left(x,|u|^{2}\right)\right) d x$, then we can write $i h^{\alpha}(x, t) \frac{\partial u}{\partial t^{\alpha}}=\frac{\delta H_{t}}{\delta u}$, where $\frac{\delta}{\delta u}=\frac{\partial}{\partial u}-D_{x} \frac{\partial}{\partial u_{x}}$ is the Euler-Lagrange operator, and $f=\frac{\partial g}{\partial|u|^{2}}$. 
(e) The solutions of multitime Hartree PDE

$$
i h^{\alpha}(x, t) \frac{\partial u}{\partial t^{\alpha}}+\Delta u=\left( \pm|x|^{n}|u|^{2}\right) u
$$

and the solutions of multitime logarithmic Schrödinger PDE (superfluids, quantum gravity)

$$
i h^{\alpha}(x, t) \frac{\partial u}{\partial t^{\alpha}}+\Delta u+u \ln |u|^{2}=0
$$

can be studied by procedures similar to those used for the multitime NLSE.

The multitime NLSE has been indicated to manage the evolution of a multitime wave packet in a weakly nonlinear and dispersive medium and has possible applications in diverse fields such as nonlinear optics, water waves and plasma physics. In this paper, it is shown that the multitime NLSE solutions can be expressed analytically by specific methods.

Section 2 analyzes solutions of a special multitime NLSE obtained by fixing a multitemporal direction $h$. Section 3 introduces and studies solutions of the multitime NLSE with a specified $f$. Section 4 gives solutions of multitime NLSE whose argument is independent of $x$. Section 4.1 refers to first integral theory in the context of multitime NLSE. Section 4.2 gives a theorem about the solutions of multitime NLSE with the argument independent of $x$. Section 5 introduces the multitime NLSE in a Riemannian setting. Section 6 formulates some conclusions that underline the authors' thoughts.

The recent mathematical literature dedicated to the context insists on the following topics: The papers [2-5] give properties of nonlinear Schrödinger equation; [6,7] underline an exact solution of the single-time Schrödinger equation; [8] introduces and studies the multitime Schrödinger equation; [1,9-12] introduce and describe the multitime solitons as solutions of multitime PDEs. The paper [13] refers to solving the time-dependent Schrödinger equation via Laplace transform on $t$. A single-time Schrödinger equation in Riemannian setting is studied in the paper [14]. The paper [15] comes closest to our style by offering "methods for constructing complex solutions of nonlinear PDEs using simpler solutions".

For possible numerical solutions we can use the techniques from our papers [16-19], using either discretization with respect to space variable $x$, or in relation to multitime variable $t$, or both.

\section{Solutions of a Special Multitime NLSE}

We will consider a particular form of the Equation (1), assuming that the vector field $h=\left(h^{\alpha}\right)$ does not depend on $x$, and the function $f$ and the vector field $h$ are of class $\mathcal{C}^{1}$. Consequently, in this case the multitime NLSE has the form

$$
i h^{\alpha}(t) \frac{\partial u}{\partial t^{\alpha}}+\frac{\partial^{2} u}{\partial x^{2}}+f\left(x,|u|^{2}\right) u=0 .
$$

Definition 1. Let $I_{0} \subseteq I_{1}$ be an interval and let $D_{0} \subseteq D$ be an open subset. A function $u: I_{0} \times D_{0} \rightarrow \mathbb{C}$ is called exact solution of the multitime NLSE if: $(i)$ it is of class $\mathcal{C}^{1}$; (ii) there exists its partial derivative of the second order with respect to $x$; (iii) we have $|u(x, t)|^{2} \in I_{2}$ for any $(x, t)=\left(x, t^{1}, t^{2}, \ldots, t^{m}\right) \in I_{0} \times D_{0}$; (iv) and $u$ verifies the relation (2) on $I_{0} \times D_{0}$ (generally, the solutions are not globally defined).

Note that from the relationship (2) it follows that the function $\frac{\partial^{2} u}{\partial x^{2}}$ is continuous.

Suppose $m \geq 2$. Let us give some methods to find solutions of multitime NLSE (2), using the orbits of the vector field $h$.

The symmetric ODEs system

$$
\frac{\mathrm{d} t^{1}}{h^{1}(t)}=\frac{\mathrm{d} t^{2}}{h^{2}(t)}=\ldots=\frac{\mathrm{d} t^{m}}{h^{m}(t)}
$$


describes the orbits of the vector field $h=\left(h^{\alpha}\right)$. The first integrals of this ODEs system are $\mathcal{C}^{1}$ solutions of the PDE

$$
h^{\alpha}(t) \frac{\partial w}{\partial t^{\alpha}}(t)=0
$$

Remark 2. (i) Along each trajectory of the ODEs system $\frac{d}{d \tau} t(\tau)=h(t(\tau))$, we have $h^{\alpha} \frac{\partial u}{\partial t^{\alpha}}=\frac{d t^{\alpha}}{d \tau} \frac{\partial u}{\partial t^{\alpha}}=\frac{d u}{d \tau}$.

(ii) Let us consider $r \in \mathbb{N}^{*}$, and $\Omega \subseteq D, U \subseteq \mathbb{R}^{r}$ as open subsets. Suppose that $\Psi_{1}, \Psi_{2}$, $\ldots \Psi_{r}: \Omega \rightarrow \mathbb{R}$ are first integrals of ODEs system (3) such that $\left(\Psi_{1}(t), \Psi_{2}(t), \ldots, \Psi_{r}(t)\right) \in U$, for all $t \in \Omega$. Let $E: U \rightarrow \mathbb{R}$ be an arbitrary $\mathcal{C}^{1}$ function. Then, the function $\Psi: \Omega \rightarrow \mathbb{R}$, $\Psi(t)=E\left(\Psi_{1}(t), \Psi_{2}(t), \ldots, \Psi_{r}(t)\right)$ is a first integral for the ODEs system (3).

Remark 3. Let us choose $t_{0} \in D$, for which there exists $\alpha_{0} \in\{1,2, \ldots, m\}$, such that $h^{\alpha_{0}}\left(t_{0}\right) \neq 0$.

Suppose that $F_{1}, F_{2}, \ldots, F_{m-1}: V \rightarrow \mathbb{R}$ are $\mathcal{C}^{1}$ functional independent (see the condition (40)) first integrals of the $O D E s$ system (3), where $V \subseteq D, t_{0} \in V, V$ is open and connected such that for all $t \in V$, we have $h^{\alpha_{0}}(t) \neq 0$. These first integrals determine the vector field $F(t):=\left(F_{1}(t), F_{2}(t), \ldots, F_{m-1}(t)\right), t \in V$.

Any other first integral of ODEs system (3), defined in a neighborhood of $t_{0}$, has the form $\Psi(t)=E(F(t))$, for all $t \in W_{0}$, where $E: U \rightarrow \mathbb{R}$ is a $\mathcal{C}^{1}$ function, $U \subseteq \mathbb{R}^{m-1}, U$ open; $t_{0} \in W_{0} \subseteq V, W_{0}$ open; $F(t) \in U$, for all $t \in W_{0}$ (see the Proposition 11 and the other results of the Section 4.1).

Proposition 1. Let $k \in \mathbb{R}$ be a fixed constant and the subset $D_{0} \subseteq D$ be open.

(a) Suppose that the $\mathcal{C}^{1}$ function $v: D_{0} \rightarrow \mathbb{C}$ is a solution of the PDE

$$
i h^{\alpha}(t) \frac{\partial v}{\partial t^{\alpha}}(t)=k v(t)
$$

such that $|v(t)|=1$, for any $t \in D_{0}$. If the function $\varphi: I_{0} \times D_{0} \rightarrow \mathbb{C}\left(I_{0}\right.$ subinterval of $\left.I_{1}\right)$ is a solution of a multitime NLSE

$$
i h^{\alpha}(t) \frac{\partial \varphi}{\partial t^{\alpha}}+\frac{\partial^{2} \varphi}{\partial x^{2}}+\left(k+f\left(x,|\varphi|^{2}\right) \varphi=0,\right.
$$

then the function $u: I_{0} \times D_{0} \rightarrow \mathbb{C}$,

$$
u(x, t)=\varphi(x, t) v(t), \quad \text { for all }(x, t) \in I_{0} \times D_{0},
$$

is a solution of a multitime NLSE (2).

(b) Suppose that the $\mathcal{C}^{1}$ function $\omega_{0}: D_{0} \rightarrow \mathbb{R}$ verifies the PDE $h^{\alpha}(t) \frac{\partial \omega_{0}}{\partial t^{\alpha}}(t)=1$, for any $t \in D_{0}$ (the local description of functions of type $\omega_{0}$ is found in Proposition 10 , selecting $q=1$ ). Then the function $v: D_{0} \rightarrow \mathbb{C}, v(t)=e^{-i k \omega_{0}(t)}$, for all $t \in D_{0}$, is a $\mathcal{C}^{1}$ solution of the PDE (5), with $|v(t)|=1$, for all $t \in D_{0}$.

If there is an index $\beta \in\{1,2, \ldots, m\}$ for which the component $h^{\beta}$ depends only on the variable $t^{\beta}$ and $h^{\beta}\left(t^{\beta}\right) \neq 0$, for any $t^{\beta}$, and if $H$ is a primitive of the function $\frac{1}{h^{\beta}}$, then we can select $\omega_{0}(t):=H\left(t^{\beta}\right)$.

Proof. (a) Replacing the function $u$ in (2), we find

$$
\begin{gathered}
i h^{\alpha}(t) \frac{\partial \varphi}{\partial t^{\alpha}}(x, t) \cdot v(t)+\varphi(x, t) \cdot i h^{\alpha}(t) \frac{\partial v}{\partial t^{\alpha}}(t) \\
+\frac{\partial^{2} \varphi}{\partial x^{2}}(x, t) \cdot v(t)+f\left(x,|\varphi(x, t)|^{2}\right) \cdot \varphi(x, t) \cdot v(t)=0 .
\end{gathered}
$$

Since the function $v(\cdot)$ is a solution of PDE (5), it turns out that the above equality is equivalent to 


$$
\begin{aligned}
i h^{\alpha}(t) \frac{\partial \varphi}{\partial t^{\alpha}}(x, t) \cdot v(t)+k \varphi(x, t) \cdot v(t) \\
\quad+\frac{\partial^{2} \varphi}{\partial x^{2}}(x, t) \cdot v(t)+f\left(x,|\varphi(x, t)|^{2}\right) \cdot \varphi(x, t) \cdot v(t)=0,
\end{aligned}
$$

and this last equality is true since the function $\varphi$ is a solution of the PDE (6).

(b) The statements are verified immediately by direct calculation.

Proposition 2. Let $\varphi: I_{0} \times D_{0} \rightarrow \mathbb{C}$ be a solution of multitime NLSE (2) ( $I_{0}$ subinterval of $I_{1}$; $D_{0} \subseteq D, D_{0}$ open). Let $\Psi: D_{0} \rightarrow \mathbb{R}$ be a $\mathcal{C}^{1}$ first integral of the ODEs system (3).

Then, the function $u: I_{0} \times D_{0} \rightarrow \mathbb{C}$,

$$
u(x, t)=\varphi(x, t) e^{i \Psi(t)}, \quad \text { for all }(x, t) \in I_{0} \times D_{0},
$$

is a solution of multitime NLSE (2).

Proof. We apply Proposition 1 , for $k=0$. If $k=0$, then the PDE (6) becomes the PDE (2). One verifies automatically that the function $v(\cdot)=e^{i \Psi(\cdot)}$ is a solution of the PDE (5), for $k=0$. More $|v(t)|=1$, for all $t \in D_{0}$.

Corollary 1. Suppose that the function $f$ does not depend on $x$, i.e., there exists the $\mathcal{C}^{1}$ function $\widetilde{f}: I_{2} \rightarrow \mathbb{R}$, such that $f(x, p)=\widetilde{f}(p)$, for all $x \in \mathbb{R}$, for all $p \in I_{2}$ (we have $\left.I_{1}=\mathbb{R}\right)$. Let $p_{0} \in I_{2}$, with $\widetilde{f}\left(p_{0}\right)=0$. Let $\Psi: D_{0} \rightarrow \mathbb{R}$ be a $\mathcal{C}^{1}$ first integral of ODEs system (3) $\left(D_{0} \subseteq D, D_{0}\right.$ open).

Then, the function $u: \mathbb{R} \times D_{0} \rightarrow \mathbb{C}, u(x, t)=\sqrt{p_{0}} \cdot e^{i \Psi(t)}, \quad$ for all $(x, t) \in \mathbb{R} \times D_{0}$, is a solution of multitime NLSE (2).

Proof. The constant function $\varphi(x, t):=\sqrt{q_{0}}$ is a solution of PDE (2). The conclusion results immediately by applying Proposition 2 .

Proposition 3. Let $k \in \mathbb{R}$ be a constant and let $v: D_{0} \rightarrow \mathbb{C}$ be a $\mathcal{C}^{1}$ solution of PDE (5), such that $|v(t)|=1$, for any $t \in D_{0}\left(D_{0} \subseteq D, D_{0}\right.$ open $)$.

Let us consider $\varphi(x, t, c)$, with $c=\left(c_{1}, \ldots, c_{r}\right) \in G$, a solution of the PDE (6), which depends on $r$ parameters, where $G$ is an open subset of $\mathbb{R}^{r}$.

Hypotheses: the function $\varphi: I_{0} \times D_{0} \times G \rightarrow \mathbb{C}$ is of class $\mathcal{C}^{1}$ with respect to all arguments; there exists $\frac{\partial^{2} \varphi}{\partial x^{2}}$ and it is continuous with respect to all arguments; furthermore, for each $c \in G$, the function $\varphi(\cdot, \cdot \cdot, c): I_{0} \times D_{0} \rightarrow \mathbb{C}$ is a solution of the multitime NLSE (6) ( $I_{0}$ subinterval of $\left.I_{1}\right)$.

Suppose that the $\mathcal{C}^{1}$ functions $\Psi_{1}, \Psi_{2}, \ldots, \Psi_{r}, \Psi_{r+1}: D_{0} \rightarrow \mathbb{R}$ are first integrals of the ODEs system (3), such that, for any $t \in D_{0}$, to have $\left(\Psi_{1}(t), \ldots, \Psi_{r}(t)\right) \in G$.

Then, the function $u: I_{0} \times D_{0} \rightarrow \mathbb{C}$,

$$
u(x, t)=\varphi\left(x, t, \Psi_{1}(t), \ldots, \Psi_{r}(t)\right) \cdot v(t) \cdot e^{i \Psi_{r+1}(t)}, \quad \text { for all }(x, t) \in I_{0} \times D_{0},
$$

is a solution of the multitime NLSE (2).

Proof. Let $\widetilde{\varphi}(x, t):=\varphi\left(x, t, \Psi_{1}(t), \ldots, \Psi_{r}(t)\right)$. We check that $\widetilde{\varphi}$ is a solution of the PDE (6). Denote $\Phi(t)=\left(\Psi_{1}(t), \ldots, \Psi_{r}(t)\right)$. One substitutes $\widetilde{\varphi}$ in (6) and one obtains

equivalent to

$$
\begin{aligned}
i h^{\alpha}(t) & \sum_{j=1}^{r} \frac{\partial \varphi}{\partial c_{j}}(x, t, \Phi(t)) \cdot \frac{\partial \Psi_{j}}{\partial t^{\alpha}}(t)+i h^{\alpha}(t) \frac{\partial \varphi}{\partial t^{\alpha}}(x, t, \Phi(t)) \\
& +\frac{\partial^{2} \varphi}{\partial x^{2}}(x, t, \Phi(t))+\left(k+f\left(x,|\varphi(x, t, \Phi(t))|^{2}\right)\right) \cdot \varphi(x, t, \Phi(t))=0
\end{aligned}
$$

$$
\begin{aligned}
i \sum_{j=1}^{r} \frac{\partial \varphi}{\partial c_{j}}(x, t, \Phi(t)) \cdot h^{\alpha}(t) \frac{\partial \Psi_{j}}{\partial t^{\alpha}}(t)+i h^{\alpha}(t) \frac{\partial \varphi}{\partial t^{\alpha}}(x, t, \Phi(t)) \\
\quad+\frac{\partial^{2} \varphi}{\partial x^{2}}(x, t, \Phi(t))+\left(k+f\left(x,|\varphi(x, t, \Phi(t))|^{2}\right)\right) \cdot \varphi(x, t, \Phi(t))=0
\end{aligned}
$$


which is true because functions $\Psi_{j}$ are first integrals, and $\varphi(\cdot, \cdot, c)$ verifies the relation (6) for any $c \in G$, so also for $c=\Phi(t)$.

In this way we showed that $\widetilde{\varphi}$ is a solution of PDE (6). According to Proposition 1, we find that $\widetilde{\varphi}(x, t) \cdot v(t)$ is a solution of PDE (2). Due to Proposition 2, it follows that $\widetilde{\varphi}(x, t) \cdot v(t) \cdot e^{i \Psi_{r+1}(t)}$ is a solution of the PDE (2).

Proposition 4. Suppose that $G$ is an open subset of $\mathbb{R}^{r}$. Let us consider $\varphi(x, t, c)$, with $c=\left(c_{1}, \ldots, c_{r}\right) \in G$, as a solution of the PDE (2) which depends on $r$ parameters.

Hypotheses: the function $\varphi: I_{0} \times D_{0} \times G \rightarrow \mathbb{C}$ is of class $\mathcal{C}^{1}$ with respect to whole arguments; there exists $\frac{\partial^{2} \varphi}{\partial x^{2}}$, continuous in all arguments; more, for each $c \in G$, the function $\varphi(\cdot, \cdot, c)$ : $I_{0} \times D_{0} \rightarrow \mathbb{C}$ is a solution of multitime NLSE (2) ( $D_{0} \subseteq D, D_{0}$ open; $I_{0}$ subinterval of $\left.I_{1}\right)$.

Suppose $\Psi_{1}, \Psi_{2}, \ldots, \Psi_{r+1}: D_{0} \rightarrow \mathbb{R}$ are $\mathcal{C}^{1}$ first integrals of the ODEs system (3), such that, for any $(x, t) \in I_{0} \times D_{0}$, to have $\left(\Psi_{1}(t), \ldots, \Psi_{r}(t)\right) \in G$.

Then, the function $u: I_{0} \times D_{0} \rightarrow \mathbb{C}$,

$$
u(x, t)=\varphi\left(x, t, \Psi_{1}(t), \ldots, \Psi_{r}(t)\right) \cdot e^{i \Psi_{r+1}(t)}, \quad \text { for all }(x, t) \in I_{0} \times D_{0},
$$

is a solution of multitime NLSE (2).

Proof. One applies Proposition 3 for $k=0$ and $v(t)=1$.

Now let us use an original ODE that incorporates the De Boer-Ludford ODE in plasma physics and the Stuart-Landau ODE in hydrodynamic stability.

Proposition 5. Let us consider the second order differential equation

$$
y^{\prime \prime}+\left(k+f\left(x, y^{2}\right)\right) y=0,
$$

where $k$ is a real fixed number (y real solution).

Let $\xi\left(\cdot, c_{1}, \ldots, c_{r}\right): I_{0} \rightarrow \mathbb{R}$ be a solution of the ODE (8), which depends on $r$ parameters, with $\left(c_{1}, \ldots, c_{r}\right) \in G$, where $G$ is an open subset of $\mathbb{R}^{r}$ and $I_{0}$ is a subinterval of $I_{1}$. Suppose that the function $\xi: I_{0} \times G \rightarrow \mathbb{R}$ is of class $\mathcal{C}^{1}$ with respect to all arguments.

Assume that the $\mathcal{C}^{1}$ function $v: D_{0} \rightarrow \mathbb{C}$ is solution of PDE (5), such that, $|v(t)|=1$, for any $t \in D_{0}\left(D_{0} \subseteq D, D_{0}\right.$ open $)$.

If the $\mathcal{C}^{1}$ functions $\Psi_{1}, \Psi_{2}, \ldots, \Psi_{r}, \Psi_{r+1}: D_{0} \rightarrow \mathbb{R}$ are first integrals for ODEs system (3), such that, for any $t \in D_{0}$, to have $\left(\Psi_{1}(t), \ldots, \Psi_{r}(t)\right) \in G$, then, the function $u: I_{0} \times D_{0} \rightarrow \mathbb{C}$,

$$
u(x, t)=\xi\left(x, \Psi_{1}(t), \ldots, \Psi_{r}(t)\right) \cdot v(t) \cdot e^{i \Psi_{r+1}(t)}, \quad \text { for all }(x, t) \in I_{0} \times D_{0},
$$

is a solution of multitime NLSE (2).

Proof. Let $\varphi: I_{0} \times D_{0} \times G \rightarrow \mathbb{C}, \varphi(x, t, c):=\xi(x, c)$, for all $(x, t, c) \in I_{0} \times D_{0} \times G$. One observes immediately that, for all $c=\left(c_{1}, \ldots, c_{r}\right) \in G$, the function $\varphi(\cdot, \cdot, c)$ is a solution of the PDE (6). One applies Proposition 3 and we obtain the conclusion.

Applying Proposition 5, for $k=0, v=1$, we find the next result.

Proposition 6. Let us consider the second order ODE

$$
y^{\prime \prime}+f\left(x, y^{2}\right) y=0
$$

Impose $\xi\left(\cdot, c_{1}, \ldots, c_{r}\right)$ as a solution of the Equation (9), which depends on $r$ parameters, $\xi\left(\cdot, c_{1}, \ldots, c_{r}\right): I_{0} \rightarrow \mathbb{R}$; with $\left(c_{1}, \ldots, c_{r}\right) \in G$; where $G$ is an open subset of $\mathbb{R}^{r}$ and $I_{0}$ is a subinterval of $I_{1}$. Suppose that the function $\xi: I_{0} \times G \rightarrow \mathbb{R}$ is of class $\mathcal{C}^{1}$ with respect to all arguments. 
If the $\mathcal{C}^{1}$ functions $\Psi_{1}, \Psi_{2}, \ldots, \Psi_{r}, \Psi_{r+1}: D_{0} \rightarrow \mathbb{R}$ are first integrals for the ODEs system (3), such that, for any $t \in D_{0}$, to have $\left(\Psi_{1}(t), \ldots, \Psi_{r}(t)\right) \in G$, then the function $u: I_{0} \times D_{0} \rightarrow \mathbb{C}$,

$$
u(x, t)=\xi\left(x, \Psi_{1}(t), \ldots, \Psi_{r}(t)\right) \cdot e^{i \Psi_{r+1}(t)}, \quad \text { for all }(x, t) \in I_{0} \times D_{0},
$$

is a solution of multitime NLSE (2).

According to Remark 3, each of the first integrals $\Psi_{j}$, which appears in Propositions 2-6, has locally the form $\Psi_{j}(t)=E_{j}(F(t))$, with $F(t)=\left(F_{1}(t), F_{2}(t), \ldots, F_{m-1}(t)\right)$, where $F_{1}, F_{2}$, $\ldots, F_{m-1}$ are functional independent first integrals for the ODEs system (3).

Remark 4. Suppose that $I_{1}$ is an open interval; let $I_{2}=(\mu, \infty)$, with $\mu \geq 0$; let $I_{3}=(\sqrt{\mu}, \infty)$.

For $k \in \mathbb{R}, \lambda \in I_{1}, c_{1} \in I_{3}, c_{2} \in \mathbb{R}$, let $\sigma\left(\cdot, \lambda ; c_{1}, c_{2} ; k\right)$ be the maximal solution $y(\cdot)$ of Equation (8) which verifies $y(\lambda)=c_{1}$ and $y^{\prime}(\lambda)=c_{2}$. The domain of definition of this solution is an open interval $I\left(\lambda ; c_{1}, c_{2} ; k\right) \subseteq I_{1}$, with $\lambda \in I\left(\lambda ; c_{1}, c_{2} ; k\right)$; the codomain is $I_{3}$.

The set $M:=\left\{\left(x, \lambda ; c_{1}, c_{2} ; k\right) \mid \lambda \in I_{1}, c_{1} \in I_{3}, c_{2} \in \mathbb{R}, k \in \mathbb{R}, x \in I\left(\lambda ; c_{1}, c_{2} ; k\right)\right\}$ is open. The function $\sigma: M \rightarrow I_{3}$ is of class $\mathcal{C}^{1}$; the function $\frac{\partial \sigma}{\partial x}: M \rightarrow I_{3}$ is of class $\mathcal{C}^{1}$. We have: $\sigma\left(\lambda, \lambda ; c_{1}, c_{2} ; k\right)=c_{1}$ and $\frac{\partial \sigma}{\partial x}\left(\lambda, \lambda ; c_{1}, c_{2} ; k\right)=c_{2}$.

Let $\left(x_{0}, t_{0}\right) \in I_{1} \times D$. Suppose there exists $\alpha_{0} \in\{1,2, \ldots, m\}$, such that $h^{\alpha_{0}}\left(t_{0}\right) \neq 0$. Let $F_{1}, F_{2}, \ldots, F_{m-1}: V \rightarrow \mathbb{R}$ be $\mathcal{C}^{1}$ first integrals for the ODEs system (3), with $V \subseteq D, t_{0} \in V, V$ open, such that for all $t \in V$, the condition (40) is met.

We introduce the vector field $F(t)=\left(F_{1}(t), F_{2}(t), \ldots, F_{m-1}(t)\right), t \in V$.

Let $c_{1,0} \in I_{3}, c_{2,0} \in \mathbb{R}, k_{0} \in \mathbb{R}$. Since $M$ is an open set, it turns out that there exists open intervals $I_{0}, L_{0}, L_{1}, L_{2}, L_{3}$, such that $x_{0} \in I_{0}, x_{0} \in L_{0}, c_{1,0} \in L_{1}, c_{2,0} \in L_{2}, k_{0} \in L_{3}$, and $I_{0} \times L_{0} \times L_{1} \times L_{2} \times L_{3} \subseteq M$.

We need a $\mathcal{C}^{1}$ function $v: D_{0} \times \mathbb{R} \rightarrow \mathbb{C},\left(t_{0} \in D_{0} \subseteq D, D_{0}\right.$ open $)$, with the properties:

- for all $k \in \mathbb{R}$, the function $v(\cdot ; k): D_{0} \rightarrow \mathbb{C}$ is a solution of the PDE (5);

- for all $k \in \mathbb{R}$, for all $t \in D_{0}$, we have $|v(t ; k)|=1$ (for example, $v$ can have the form in Proposition $1, b)$, i.e., $v(t ; k)=e^{-i k \omega_{0}(t)}$, with $\omega_{0}: D_{0} \rightarrow \mathbb{R}$, of class $\mathcal{C}^{1}$, which verifies $\left.h^{\alpha}(t) \frac{\partial \omega_{0}}{\partial t^{\alpha}}(t)=1\right)$.

We add the $\mathcal{C}^{1}$ functions $E_{1}: U_{0} \rightarrow L_{1}, E_{2}: U_{0} \rightarrow L_{2}, E_{3}: U_{0} \rightarrow \mathbb{R}, E_{4}: U_{0} \rightarrow L_{3}$ $\left(U_{0} \subseteq \mathbb{R}^{m-1}, U_{0}\right.$ open); let $W_{0} \subseteq D_{0} \cap V, W_{0}$ an open neighborhood of $t_{0}$, such that, for any $t \in W_{0}$ to have $F(t) \in U_{0}$.

We use the auxiliary function $\xi: I_{0} \times L_{1} \times L_{2} \times L_{3} \rightarrow I_{3}, \xi\left(x, c_{1}, c_{2} ; k\right)=\sigma\left(x, x_{0} ; c_{1}, c_{2} ; k\right)$, for all $\left(x, c_{1}, c_{2} ; k\right) \in I_{0} \times L_{1} \times L_{2} \times L_{3}$.

For $k \in L_{3}$ fixed, the function $\xi\left(\cdot, c_{1}, c_{2} ; k\right)$ is a solution of PDE (8) which depends on two parameters $\left(c_{1}, c_{2}\right) \in L_{1} \times L_{2}$ and has the properties of hypothesis of Proposition 5 . From Proposition 5 it follows that the function

$$
\xi\left(x, E_{1}(F(t)), E_{2}(F(t)) ; k\right) \cdot v(t ; k) \cdot e^{i E_{3}(F(t))}\left(\text { with }(x, t) \in I_{0} \times W_{0}\right)
$$

is a solution of PDE (2); but this solution also depends on one parameter, namely by $k$. From Proposition 4 (with $r=1$, choosing $\Psi_{r+1}=0$ ) it follows that the function $u: I_{0} \times W_{0} \rightarrow \mathbb{C}$, defined for all $(x, t) \in I_{0} \times W_{0}$ by

$$
u(x, t)=\xi\left(x, E_{1}(F(t)), E_{2}(F(t)) ; E_{4}(F(t))\right) \cdot v\left(t ; E_{4}(F(t)) \cdot e^{i E_{3}(F(t))}\right.
$$

is a solution of multitime NLSE (2).

Lemma 1. Suppose that the function $f$ does not depend on $x$, i.e., there exists a $\mathcal{C}^{1}$ function $\widetilde{f}: I_{2} \rightarrow \mathbb{R}$, such that $f(x, p)=\widetilde{f}(p)$, for all $x \in \mathbb{R}$, for all $p \in I_{2}$ (we have $I_{1}=\mathbb{R}$ ). Let $c_{1}, c_{2} \in \mathbb{R}$, such that $c_{1}^{2} \in I_{2} ;$ let $k:=c_{2}^{2}-\widetilde{f}\left(c_{1}^{2}\right)$.

(a) The function $y: \mathbb{R} \rightarrow \mathbb{C}, y(x)=c_{1} e^{i c_{2} x}$, for all $x \in \mathbb{R}$, is a solution of the ODE

$$
y^{\prime \prime}+\left(k+\widetilde{f}\left(|y|^{2}\right)\right) y=0 .
$$


(b) If $c_{2}^{2}=\widetilde{f}\left(c_{1}^{2}\right)$, then the function $u: \mathbb{R} \times D \rightarrow \mathbb{C}, u(x, t)=c_{1} e^{i c_{2} x}$, for all $x \in \mathbb{R}$, for all $t \in D$, is a solution of multitime NLSE (1).

Proof. (a) Replacing $y$ in the equality (10) one obtains $-c_{2}^{2} \cdot c_{1} e^{i c_{2} x}+\left(k+\widetilde{f}\left(c_{1}^{2}\right)\right) \cdot c_{1} e^{i c_{2} x}=0$, which is true because $k=c_{2}^{2}-\widetilde{f}\left(c_{1}^{2}\right)$.

(b) A similar calculation is made with the one from the point $a)$, with $k=0$.

Proposition 7. Let us consider the multitime NLSE (2); suppose there exists the $\mathcal{C}^{1}$ function $\widetilde{f}: I_{2} \rightarrow \mathbb{R}$, such that $f(x, p)=\widetilde{f}(p)$, for all $x \in \mathbb{R}$, for all $p \in I_{2}$ (in this case we have $I_{1}=\mathbb{R}$ ). Let $I_{3} \subseteq I_{2}, I_{3}$ interval, such that, for all $c_{1} \in I_{3}$ to have $c_{1}^{2} \in I_{2}$.

Let $D_{0} \subseteq D, D_{0}$ open; let the $\mathcal{C}^{1}$ function $v: D_{0} \times \mathbb{R} \rightarrow \mathbb{C}$, with the properties: - for each $k \in \mathbb{R}$, the function $v(\cdot ; k): D_{0} \rightarrow \mathbb{C}$ is a solution of the Equation (5);

- for all $t \in D_{0}$, for all $k \in \mathbb{R}$, we have $|v(t ; k)|=1$ (for example $v$ might have the form indicated in Proposition 1, $(b)$, i.e., $v(t ; k)=e^{-i k \omega_{0}(t)}$, with $\omega_{0}: D_{0} \rightarrow \mathbb{R}$, of class $\mathcal{C}^{1}$, which verifies $\left.h^{\alpha}(t) \frac{\partial \omega_{0}}{\partial t^{\alpha}}(t)=1\right)$.

Suppose the $\mathcal{C}^{1}$ functions $\Psi_{1}: D_{0} \rightarrow I_{3}$ and $\Psi_{2}, \Psi_{3}: D_{0} \rightarrow \mathbb{R}$ are first integrals for the ODEs system (3).

Then, the function $u: \mathbb{R} \times D_{0} \rightarrow \mathbb{C}$ defined for all $(x, t) \in \mathbb{R} \times D_{0}$ by the formula

$$
u(x, t)=\Psi_{1}(t) e^{i x \Psi_{2}(t)+i \Psi_{3}(t)} v\left(t ;\left(\Psi_{2}(t)\right)^{2}-\widetilde{f}\left(\left(\Psi_{1}(t)\right)^{2}\right)\right),
$$

is a solution of the multitime NLSE (2).

Proof. Let $w\left(x, t ; c_{1}, c_{2}\right):=c_{1} e^{i c_{2} x}$, for all $x \in \mathbb{R}, t \in D_{0}, c_{1} \in I_{3}, c_{2} \in \mathbb{R}$.

From Lemma 1, (a), it follows that the function $w\left(\cdot, \cdot ; c_{1}, c_{2}\right)$ is a solution of the Equation (6), with $k=c_{2}^{2}-\widetilde{f}\left(c_{1}^{2}\right)$.

Let $\varphi\left(x, t ; c_{1}, c_{2}\right):=w\left(x, t ; c_{1}, c_{2}\right) v\left(t ; c_{2}^{2}-\widetilde{f}\left(c_{1}^{2}\right)\right)$, for all $x \in \mathbb{R}, t \in D_{0}, c_{1} \in I_{3}, c_{2} \in \mathbb{R}$. From Proposition 1 it follows that $\varphi\left(\cdot, \cdot ; c_{1}, c_{2}\right)$ is a solution of multitime NLSE (2); the function $\varphi\left(\cdot, \cdot ; c_{1}, c_{2}\right)$ depends obviously on two parameters. The function $\varphi\left(\cdot, \cdot ; c_{1}, c_{2}\right)$ satisfies the conditions in Proposition 4, with $r=2$. One applies Proposition 4 and the conclusion immediately follows.

Lemma 2. Let $k \in \mathbb{R}$ be a constant and let $g_{1}: I_{0} \rightarrow \mathbb{R} \backslash\{0\}$ (with $I_{0}$ subinterval of $I_{1}$ ) be a solution of the $O D E$

$$
g_{1}^{\prime \prime}-\frac{k^{2}}{g_{1}^{3}}+\left(k+f\left(x, g_{1}^{2}\right)\right) g_{1}=0 .
$$

Let $g_{2}: I_{0} \rightarrow \mathbb{R}$ be a primitive of the function $\frac{1}{g_{1}^{2}}$. Then, the function

$y: I_{0} \rightarrow \mathbb{C}, \quad y(x)=g_{1}(x) e^{i k g_{2}(x)}, \quad$ for all $x \in I_{0}$, is a solution of the equation

$$
y^{\prime \prime}+\left(k+f\left(x,|y|^{2}\right)\right) y=0 .
$$

Proof. $y^{\prime}(x)=g_{1}^{\prime}(x) e^{i k g_{2}(x)}+i k g_{1}(x) g_{2}^{\prime}(x) e^{i k g_{2}(x)}=g_{1}^{\prime}(x) e^{i k g_{2}(x)}+\frac{i k}{g_{1}(x)} e^{i k g_{2}(x)}$;

$$
\begin{aligned}
& y^{\prime \prime}(x)=g_{1}^{\prime \prime}(x) e^{i k g_{2}(x)}+i k g_{1}^{\prime}(x) g_{2}^{\prime}(x) e^{i k g_{2}(x)}-\frac{i k g_{1}^{\prime}(x)}{\left(g_{1}(x)\right)^{2}} e^{i k g_{2}(x)}-\frac{k^{2} g_{2}^{\prime}(x)}{g_{1}(x)} e^{i k g_{2}(x)} \\
= & g_{1}^{\prime \prime}(x) e^{i k g_{2}(x)}-\frac{k^{2}}{\left(g_{1}(x)\right)^{3}} e^{i k g_{2}(x)} ; \\
& y^{\prime \prime}(x)+\left(k+f\left(x,|y(x)|^{2}\right)\right) y(x) \\
= & g_{1}^{\prime \prime}(x) e^{i k g_{2}(x)}-\frac{k^{2}}{\left(g_{1}(x)\right)^{3}} e^{i k g_{2}(x)}+\left(k+f\left(x,\left(g_{1}(x)\right)^{2}\right)\right) g_{1}(x) e^{i k g_{2}(x)}=0 . \quad
\end{aligned}
$$


Proposition 8. Let $k \in \mathbb{R}$ be a constant; let $g_{1}: I_{0} \rightarrow \mathbb{R} \backslash\{0\}$ be a solution of the Equation (11); let $g_{2}: I_{0} \rightarrow \mathbb{R}$ be a primitive of the function $\frac{1}{g_{1}^{2}}\left(I_{0}\right.$ subinterval of $\left.I_{1}\right)$. Suppose the $\mathcal{C}^{1}$ function $v: D_{0} \rightarrow \mathbb{C}$ is a solution of the Equation (5), such that $|v(t)|=1$, for all $t \in D_{0}\left(D_{0} \subseteq D\right.$, $D_{0}$ open).

Then, the function $u: I_{0} \times D_{0} \rightarrow \mathbb{C}$,

$$
u(x, t)=g_{1}(x) e^{i k g_{2}(x)} v(t), \quad \text { for all }(x, t) \in I_{0} \times D_{0},
$$

is a solution of multitime NLSE (2).

Proof. According to Lemma 2, the function $y(x)=g_{1}(x) e^{i k g_{2}(x)}$ is a solution of equation (12) which is equivalent to the fact that the function $\varphi(x, t):=g_{1}(x) e^{i k g_{2}(x)}$ is a solution of the Equation (6). From Proposition 1 it follows that $u$ is a solution of multitime NLSE (2).

The function $g_{1}$ depends on two constants and the parameter $k$; the same for the function $g_{2}$. The function $v$ depends also on the parameter $k$. Using Propositions 4 and 8 , a solution can be obtained for the multitime NLSE (2) analogous to Remark 4.

\section{Multitime NLSE with a Specified $f$}

Let $c \in \mathbb{R}$ be a fixed constant. Let us consider the multitime NLSE (2), with $f: \mathbb{R} \times \mathbb{R} \rightarrow \mathbb{R}, f(x, p)=c-2 p$; i.e.,

$$
i h^{\alpha}(t) \frac{\partial u}{\partial t^{\alpha}}+\frac{\partial^{2} u}{\partial x^{2}}+\left(c-2|u|^{2}\right) u=0 .
$$

We select $t_{0} \in D$, for which it exists $\alpha_{0} \in\{1,2, \ldots, m\}$, such that $h^{\alpha_{0}}\left(t_{0}\right) \neq 0$, and the $\mathcal{C}^{1}$ functions $F_{1}, F_{2}, \ldots, F_{m-1}: V \rightarrow \mathbb{R}$, as first integrals for ODEs system (3), with $V \subseteq D$, $t_{0} \in V, V$ open, such that for all $t \in V$, the condition (40) is satisfied. We introduce the vector field $F(t)=\left(F_{1}(t), F_{2}(t), \ldots, F_{m-1}(t)\right), t \in V$.

We need the $\mathcal{C}^{1}$ function $\omega_{0}: D_{0} \rightarrow \mathbb{R}$, which for any $t \in D_{0}$ verifies $h^{\alpha}(t) \frac{\partial \omega_{0}}{\partial t^{\alpha}}(t)=1$ ( $D_{0}$ open neighborhood of $\left.t_{0}\right) ; \omega_{0}$ can be selected as in Proposition 10, $(b)$ (with $q=1$ ).

If the function $h^{\alpha_{0}}$ depends only on the variable $t^{\alpha_{0}}$ and $h^{\alpha_{0}}\left(t^{\alpha_{0}}\right) \neq 0$, for all $t^{\alpha_{0}}$, then we can select $\omega_{0}(t):=H\left(t^{\alpha_{0}}\right)$, where $H$ is a primitive of the function $\frac{1}{h^{\alpha_{0}}}$.

Let us consider the $\mathcal{C}^{1}$ functions $E_{1}, E_{2}, E_{3}: U \rightarrow \mathbb{R}$ (with $U \subseteq \mathbb{R}^{m-1}, U$ open). Suppose that for any $t \in D_{0} \cap V$ we have $F(t) \in U$, possibly replacing the domain $D_{0} \cap V$ with another smaller open neighborhood of $t_{0}$. It eventually shrinks $D_{0} \cap V$, such that the different solutions obtained in this Section, in which appear $E_{1}(F(t)), E_{2}(F(t))$ or $E_{3}(F(t))$ to be well defined (to make sense).

Concrete case: $h^{\alpha}(t)=b^{\alpha}$ constant functions, with $b^{m} \neq 0$. The PDE (13) becomes

$$
i b^{\alpha} \frac{\partial u}{\partial t^{\alpha}}+\frac{\partial^{2} u}{\partial x^{2}}+\left(c-2|u|^{2}\right) u=0 .
$$

In this case we choose $\omega_{0}(t)=\frac{t^{m}}{b^{m}}$. It is easy to see that the $m-1$ functionally independent first integrals for the ODEs system (3) can be chosen in this way

$$
F_{\gamma}(t)=b^{m} t^{\gamma}-b^{\gamma} t^{m}, \quad \text { for all } t \in \mathbb{R}^{m}, \quad \text { for all } \gamma \in\{1,2, \ldots, m-1\} .
$$

The orbits of the parallel vector field $b=\left(b^{1}, \ldots, b^{m}\right)$ are straight lines.

Lemma 3. (a) Let $a \in \mathbb{R}$ be a fixed constant. We consider the $O D E$

$$
y^{\prime \prime}=2\left(y^{2}-a^{2}\right) y
$$




$$
\text { The functions } \xi_{1}\left(x, c_{1}\right)= \pm a \frac{1-c_{1} e^{2 a x}}{1+c_{1} e^{2 a x}}, \quad \xi_{2}\left(x, c_{1}\right)=\frac{ \pm a \sqrt{2}}{\sin \left(a \sqrt{2} x+c_{1}\right)}
$$

are two solutions of the Equation (15) which depend on the parameter $c_{1}$.

(b) Let $a \in \mathbb{R}$ be a fixed constant. We consider the ODE

$$
\begin{gathered}
y^{\prime \prime}=2\left(y^{2}+a^{2}\right) y . \\
\text { The functions } \omega_{1}\left(x, c_{1}\right)= \pm a \tan \left(a x+c_{1}\right), \quad \omega_{2}\left(x, c_{1}\right)=\frac{ \pm a \sqrt{2}}{\sinh \left(a \sqrt{2} x+c_{1}\right)}
\end{gathered}
$$

are two solutions of the Equation (17) which depend on one parameter.

Let $a \in \mathbb{R}$ be an arbitrary constant. We choose $k=2 a^{2}-c$. The Equation (8) becomes the Equation (15). For this equation we found two solutions of it that depend on one parameter, namely the functions defined by the formulas (16).

Applying Proposition 5, for $v(t):=e^{-i k \omega_{0}(t)}$, we deduce that the functions

$$
\begin{aligned}
& u_{a}(x, t)= \pm a \frac{1-E_{1}(F(t)) e^{2 a x}}{1+E_{1}(F(t)) e^{2 a x}} \cdot e^{i E_{2}(F(t))+i\left(c-2 a^{2}\right) \omega_{0}(t)}, \\
& \widetilde{u}_{a}(x, t)= \pm \frac{a \sqrt{2}}{\sin \left(a \sqrt{2} x+E_{1}(F(t))\right)} \cdot e^{i E_{2}(F(t))+i\left(c-2 a^{2}\right) \omega_{0}(t)}
\end{aligned}
$$

are solutions of the Equation (13).

Now we select $k=-2 a^{2}-c$. The Equation (8) becomes (17). Two solutions of it that depend on one parameter are given by relationships (18).

So, two more families of solutions of Equation (13) are obtained:

$$
\begin{aligned}
& w_{a}(x, t)= \pm a \tan \left(a x+E_{1}(F(t))\right) \cdot e^{i E_{2}(F(t))+i\left(c+2 a^{2}\right) \omega_{0}(t)}, \\
& \widetilde{w}_{a}(x, t)= \pm \frac{a \sqrt{2}}{\sinh \left(a \sqrt{2} x+E_{1}(F(t))\right)} \cdot e^{i E_{2}(F(t))+i\left(c+2 a^{2}\right) \omega_{0}(t)} .
\end{aligned}
$$

We choose $k=-c$. The Equation (8) becomes $y^{\prime \prime}=2 y^{3}$. It is easy to see that a solution that depends on one parameter is: $\xi\left(x, c_{1}\right)= \pm \frac{1}{x+c_{1}}$.

Analogously, the functions

$$
\theta(x, t)= \pm \frac{1}{x+E_{1}(F(t))} \cdot e^{i E_{2}(F(t))+i c \omega_{0}(t)}
$$

are solutions of the Equation (13).

The functions $u_{a}, \widetilde{u}_{a}, w_{a}, \widetilde{w}_{a}$, are solutions of the Equation (13) which depend on the parameter $a$. According to Proposition 4, it follows that if we replace $a$ by $E_{3}(F(\cdot))$, we obtain again solutions of the Equation (13).

For the functions $\widetilde{u}_{a}, \widetilde{w}_{a}$, is more convenient to take $a \sqrt{2}$ as parameter; hence we will replace $a \sqrt{2}$ with $E_{3}(F(\cdot))$.

Consequently, we obtain the following solutions of the Equation (13)

$$
\begin{gathered}
u(x, t)= \pm E_{3}(F(t)) \frac{1-E_{1}(F(t)) e^{2 x E_{3}(F(t))}}{1+E_{1}(F(t)) e^{2 x E_{3}(F(t))}} \cdot e^{i E_{2}(F(t))+i\left(c-2\left(E_{3}(F(t))\right)^{2}\right) \omega_{0}(t)}, \\
\widetilde{u}(x, t)= \pm \frac{E_{3}(F(t))}{\sin \left(x E_{3}(F(t))+E_{1}(F(t))\right)} \cdot e^{i E_{2}(F(t))+i\left(c-\left(E_{3}(F(t))\right)^{2}\right) \omega_{0}(t)}, \\
\widetilde{w}(x, t)= \pm \frac{E_{3}(F(t)) \tan \left(x E_{3}(F(t))+E_{1}(F(t))\right) \cdot e^{i E_{2}(F(t))+i\left(c+2\left(E_{3}(F(t))\right)^{2}\right) \omega_{0}(t),}}{\sinh \left(x E_{3}(F(t))+E_{1}(F(t))\right)} \cdot e^{i E_{2}(F(t))+i\left(c+\left(E_{3}(F(t))\right)^{2}\right) \omega_{0}(t)},
\end{gathered}
$$

to which the solution $\theta(x, t)$ defined by the Formula (19) is added.

It is observed that we can give up writing the sign " \pm " in the above formulas, because replacing function $E_{2}(F(t))$ with function $\pi+E_{2}(F(t))$ we get the sign change $\left(e^{i \pi}=-1\right)$. 
Using Proposition 7, with $v(t ; k):=e^{-i k \omega_{0}(t)}$ (and $\widetilde{f}(p)=c-2 p$ ), the following solutions of the Equation (13)

$$
\eta(x, t)=E_{1}(F(t)) \exp \left[i x E_{2}(F(t))+i E_{3}(F(t))+i\left(c-2\left(E_{1}(F(t))\right)^{2}-\left(E_{2}(F(t))\right)^{2}\right) \omega_{0}(t)\right]
$$

are also obtained.

Lemma 4. Let $a, B$ be real constants, such that $a+B>0$ and $a \neq 0$. We consider the ODE

$$
g_{1}^{\prime \prime}-\frac{(a+B) a^{2}}{g_{1}^{3}}+\left(3 a+B-2 g_{1}^{2}\right) g_{1}=0
$$

(i) If $B<0$, then the functions

$$
g_{1,1}(x)= \pm \sqrt{a+B-B \tanh ^{2}\left(x \sqrt{-B}+c_{1}\right)}
$$

and

$$
g_{1,2}(x)= \pm \sqrt{a+B-B \operatorname{coth}^{2}\left(x \sqrt{-B}+c_{1}\right)}
$$

are solutions of the ODE (20). The functions $g_{2,1}$, respectively $g_{2,2}$ are primitives of functions $\frac{1}{g_{1,1}^{2}}$, respectively $\frac{1}{g_{1,2}^{2}}$ :

$$
\begin{aligned}
& g_{2,1}(x)=\frac{x}{a}+\frac{1}{a \sqrt{a+B}} \cdot \arctan \frac{\sqrt{-B} \tanh \left(x \sqrt{-B}+c_{1}\right)}{\sqrt{a+B}} \\
& g_{2,2}(x)=\frac{x}{a}+\frac{1}{a \sqrt{a+B}} \cdot \arctan \frac{\sqrt{-B} \operatorname{coth}\left(x \sqrt{-B}+c_{1}\right)}{\sqrt{a+B}} .
\end{aligned}
$$

(ii) If $B>0$, then the function

$$
g_{1,3}(x)= \pm \sqrt{a+B+B \tan ^{2}\left(x \sqrt{B}+c_{1}\right)}
$$

is a solution of the Equation (20). We obtain the primitive

$$
g_{2,3}(x):=\int \frac{\mathrm{d} x}{g_{1,3}(x)^{2}}=\frac{x}{a}-\frac{1}{a \sqrt{a+B}} \cdot \arctan \frac{\sqrt{B} \tan \left(x \sqrt{B}+c_{1}\right)}{\sqrt{a+B}} .
$$

(iii) If $B=0$, then the function

$$
g_{1,4}(x)= \pm \sqrt{a+\frac{1}{\left(x+c_{1}\right)^{2}}}
$$

is a solution of the Equation (20). We have

$$
g_{2,4}(x):=\int \frac{\mathrm{d} x}{g_{1,4}(x)^{2}}=\frac{x}{a}-\frac{\arctan \left(\sqrt{a}\left(x+c_{1}\right)\right)}{a \sqrt{a}} .
$$

The functions $g_{1,1}, g_{2,1}$ are well defined for any $x \in \mathbb{R}$. The functions $g_{1,2}, g_{2,2}$ are well defined for any $x \neq \frac{-c_{1}}{\sqrt{-B}}, x \in \mathbb{R}$. The functions $g_{1,3}, g_{2,3}$ are well defined for any $x \neq \frac{-c_{1}+\frac{\pi}{2}+p \pi}{\sqrt{B}}$, with $p \in \mathbb{Z}(x \in \mathbb{R})$. The functions $g_{1,4}, g_{2,4}$ are well defined for any $x \neq-c_{1}, x \in \mathbb{R}$.

The functions $g_{1,1}, g_{1,2}, g_{1,3}, g_{1,4}$ do not vanish anywhere.

Proof. The functions $g_{1,1}$ and $g_{1,2}$ are well defined since $a+B>0$ and $B<0$, hence the expressions that appear under radical in the Formulas (21) and (22) are strictly positive; one remarks immediately that also $g_{2,1}, g_{2,2}$ are well defined $(a \neq 0, a+B>0, B<0)$. 
The function $g_{1,3}$ is well defined since in this case $a+B>0$ and $B>0$, hence the expression that appears below the radical in the Formula (25) is strictly positive; it is immediately noticeable that the function $g_{2,3}$ is well defined. In case (iii), we have $B=0$ and $a+B>0$, i.e., $a>0$; we deduce that the functions $g_{1,4}$ and $g_{2,4}$ are well defined.

From the above it is immediately noticeable that the functions $g_{1,1}, g_{1,2}, g_{1,3}, g_{1,4}$ do not vanish anywhere.

We shall show that $g_{1,1}, g_{1,2}, g_{1,3}, g_{1,4}$ are solutions of the equation

$$
\left(g_{1}^{\prime}\right)^{2}=g_{1}^{4}-(3 a+B) g_{1}^{2}+3 a^{2}+2 a B-\frac{a^{3}+a^{2} B}{g_{1}^{2}},
$$

which is equivalent to (since $g_{1} \neq 0$ )

$$
\begin{gathered}
\left(g_{1} g_{1}^{\prime}\right)^{2}=g_{1}^{6}-(3 a+B) g_{1}^{4}+\left(3 a^{2}+2 a B\right) g_{1}^{2}-\left(a^{3}+a^{2} B\right) \text { or } \\
\left(g_{1} g_{1}^{\prime}\right)^{2}=\left(g_{1}^{2}-a\right)^{2}\left(g_{1}^{2}-a-B\right),
\end{gathered}
$$

so it is enough to show that $g_{1,1}, g_{1,2}, g_{1,3}, g_{1,4}$ are solutions of the Equation (30).

Case $(i)$. Denote by $T(x)$ one of the functions:

$$
T(x):=\tanh \left(x \sqrt{-B}+c_{1}\right) \text { or } \quad T(x):=\operatorname{coth}\left(x \sqrt{-B}+c_{1}\right) .
$$

In both situations we have $T^{\prime}=\sqrt{-B}\left(1-T^{2}\right)$. The relation $g_{1}^{2}=a+B-B \cdot T^{2}$ is also true; taking the derivative we obtain

$$
2 g_{1} g_{1}^{\prime}=-2 B \sqrt{-B} \cdot T\left(1-T^{2}\right) ; \quad\left(g_{1} g_{1}^{\prime}\right)^{2}=-B^{3} T^{2}\left(1-T^{2}\right)^{2} .
$$

From $g_{1}^{2}=a+B-B \cdot T^{2}$, we deduce

$$
g_{1}^{2}-a=B\left(1-T^{2}\right) \quad \text { and } \quad g_{1}^{2}-a-B=-B \cdot T^{2},
$$

hence $\left(g_{1}^{2}-a\right)^{2}\left(g_{1}^{2}-a-B\right)=B^{2}\left(1-T^{2}\right)^{2}(-B) \cdot T^{2}=\left(g_{1} g_{1}^{\prime}\right)^{2}$. The relationship (30) was obtained.

Case (ii). Denote $T(x):=\tan \left(x \sqrt{B}+c_{1}\right)$. We have $T^{\prime}=\sqrt{B}\left(1+T^{2}\right)$.

The relation $g_{1}^{2}=a+B+B \cdot T^{2}$ is also true; taking the derivative we obtain

$$
2 g_{1} g_{1}^{\prime}=2 B \sqrt{B} \cdot T\left(1+T^{2}\right) ; \quad\left(g_{1} g_{1}^{\prime}\right)^{2}=B^{3} T^{2}\left(1+T^{2}\right)^{2} .
$$

From $g_{1}^{2}=a+B+B \cdot T^{2}$, we find

$$
g_{1}^{2}-a=B\left(1+T^{2}\right) \quad \text { and } \quad g_{1}^{2}-a-B=B \cdot T^{2},
$$

hence it follows: $\left(g_{1}^{2}-a\right)^{2}\left(g_{1}^{2}-a-B\right)=B^{2}\left(1+T^{2}\right)^{2} B \cdot T^{2}=\left(g_{1} g_{1}^{\prime}\right)^{2}$. The relationship (30) was obtained.

$$
\begin{aligned}
& \text { Case }(i i i) \cdot g_{1}^{2}(x)=a+\frac{1}{\left(x+c_{1}\right)^{2}} ; 2 g_{1}(x) g_{1}^{\prime}(x)=-\frac{2}{\left(x+c_{1}\right)^{3}} \\
& \left(g_{1}(x) g_{1}^{\prime}(x)\right)^{2}=\frac{1}{\left(x+c_{1}\right)^{6}}=\left(g_{1}^{2}(x)-a\right)^{3}, \text { so even in this case the relationship }
\end{aligned}
$$
is true.

We showed that $g_{1,1}, g_{1,2}, g_{1,3}, g_{1,4}$ are solutions of the Equation (30); as we have seen, the relationship (30) is equivalently to the relation (29).

Using the derivative, from the relation (29) we obtain

$$
2 g_{1}^{\prime} g_{1}^{\prime \prime}=4 g_{1}^{3} g_{1}^{\prime}-2(3 a+B) g_{1} g_{1}^{\prime}+2 \frac{a^{3}+a^{2} B}{g_{1}^{3}} \cdot g_{1}^{\prime} .
$$

In case $(i)$, from the equality $g_{1} g_{1}^{\prime}=-B \sqrt{-B} \cdot T\left(1-T^{2}\right)$, with $T(x)=\tanh \left(x \sqrt{-B}+c_{1}\right)$ or $T(x)=\operatorname{coth}\left(x \sqrt{-B}+c_{1}\right)$, it follows that $g_{1}^{\prime}$ vanishes at a finite number of points. 
In case (ii), from equality $g_{1} g_{1}^{\prime}=B \sqrt{B} \cdot T\left(1+T^{2}\right)$, with $T(x)=\tan \left(x \sqrt{B}+c_{1}\right)$, it follows that zeros of $g_{1}^{\prime}$ are isolated points.

In case (iii), from the equality $g_{1}(x) g_{1}^{\prime}(x)=-\frac{1}{\left(x+c_{1}\right)^{3}}$, it follows that $g_{1}^{\prime}(x) \neq 0$.

Divide the relation (31) by $2 g_{1}^{\prime}(x)$, there where $g_{1}^{\prime}(x) \neq 0$; one obtains

$$
g_{1}^{\prime \prime}=2 g_{1}^{3}-(3 a+B) g_{1}+\frac{a^{3}+a^{2} B}{g_{1}^{3}} .
$$

It follows from the above that the relationship (32) is fulfilled everywhere, except for some isolated points (possibly). From continuity, it follows that the relation (32) is true throughout (on the definition domain of $g_{1}$ ).

The equality (32) is obviously equivalent to equality (20).

The relations $g_{2,1}^{\prime}(x)=\frac{1}{g_{1,1}(x)^{2}}, g_{2,2}^{\prime}(x)=\frac{1}{g_{1,2}(x)^{2}}, g_{2,3}^{\prime}(x)=\frac{1}{g_{1,3}(x)^{2}}$ and $g_{2,4}^{\prime}(x)=\frac{1}{g_{1,4}(x)^{2}}$ are verified without difficulty by direct calculation.

Furthermore, we shall determine some solutions of the Equation (13) using Proposition 8. We'll take $v(t ; k):=e^{-i k \omega_{0}(t)}$.

For each $a \in \mathbb{R}$, we consider the second order algebraic equation (with the unknown $B$ )

$$
(3 a+B-c)^{2}=(a+B) a^{2},
$$

equivalent to

$$
B^{2}-\left(a^{2}-6 a+2 c\right) B+(3 a-c)^{2}-a^{3}=0 .
$$

There exists an interval $I$ such that $0 \notin I, \frac{c}{2} \notin I$ and, for all $a \in I$, to have $(3 a-c)^{2}-a^{3}<0$.

Since $\lim _{a \rightarrow \infty}\left((3 a-c)^{2}-a^{3}\right)=-\infty$, it follows that there exists $a_{0} \geq \max \left\{0, \frac{c}{2}\right\}$, such that, for all $a \in I$, to have $(3 a-c)^{2}-a^{3}<0$. The interval can be taken $I=\left(a_{0}, \infty\right)$.

Further we consider $a \in I$.

The product of the roots of the Equation (34) is $(3 a-c)^{2}-a^{3}<0$. It follows that the roots $B_{1}(a), B_{2}(a)$ of the Equation (34), are real, distinct and have different signs. These are

$$
\begin{array}{ll}
B_{1}(a)=\frac{a^{2}-6 a+2 c-\sqrt{D(a)}}{2}<0, & \text { for all } a \in I ; \\
B_{2}(a)=\frac{a^{2}-6 a+2 c+\sqrt{D(a)}}{2}>0, & \text { for all } a \in I,
\end{array}
$$

where $D(a)=\left(a^{2}-6 a+2 c\right)^{2}-4(3 a-c)^{2}+4 a^{3}>0, a \in I$.

Let us observe that if $B$ is one of the roots of the Equation (34), then $a+B \neq 0$. If $a+B=0$, then from the equality (33) it follows $3 a+B-c=0$, i.e., $a=\frac{c}{2}$, what you cannot for $a \in I$.

Hence $a+B \neq 0$. From the relation (33), we obtain $a+B>0$.

We proved that for any $a \in I$, the relations $a+B_{1}(a)>0$ and $a+B_{2}(a)>0$ are true.

Let $a \in I$. We select $k=3 a+B_{1}(a)-c$. From equality (33) it follows $k^{2}=\left(a+B_{1}(a)\right) a^{2}$. We notice that the Equation (11) becomes in this case the Equation (20), with $B=B_{1}(a)<0$. Assumptions of Lemma $4,(i)$, are satisfied.

Let $g_{1,1}\left(x, c_{1} ; a\right)$ respectively $g_{2,1}\left(x, c_{1} ; a\right)$, the functions defined by the Formula (21), respectively (23), with $B=B_{1}(a)$.

Let $g_{1,2}\left(x, c_{1} ; a\right)$ respectively $g_{2,2}\left(x, c_{1} ; a\right)$, the functions defined by Formula (22), respectively (24), with $B=B_{1}(a)$. 
According to Lemma 4, the functions $g_{1,1}\left(\cdot, c_{1} ; a\right)$ and $g_{1,2}\left(\cdot, c_{1} ; a\right)$ are solutions of the Equation (11) and $g_{2,1}^{\prime}=\frac{1}{g_{1,1}^{2}}, g_{2,2}^{\prime}(x)=\frac{1}{g_{1,2}^{2}}$. Let us consider the functions

$$
\begin{aligned}
& \varphi_{1,1}\left(x, t, c_{1} ; a\right):=g_{1,1}\left(x, c_{1} ; a\right) e^{i\left(3 a+B_{1}(a)-c\right) g_{2,1}\left(x, c_{1} ; a\right)} v\left(t ; 3 a+B_{1}(a)-c\right), \\
& \varphi_{1,2}\left(x, t, c_{1} ; a\right):=g_{1,2}\left(x, c_{1} ; a\right) e^{i\left(3 a+B_{1}(a)-c\right) g_{2,2}\left(x, c_{1} ; a\right)} v\left(t ; 3 a+B_{1}(a)-c\right) .
\end{aligned}
$$

From Proposition 8 it follows that the functions $\varphi_{1,1}$, and $\varphi_{1,2}$ are solutions of the Equation (13), which depend on $c_{1}$ and $a$.

From Proposition 4, it follows that the functions

$$
\begin{gathered}
u_{1,1}(x, t):=g_{1,1}\left(x, E_{1}(F(t)) ; E_{2}(F(t))\right) \\
\cdot \exp \left[i\left(3 E_{2}(F(t))+B_{1}\left(E_{2}(F(t))\right)-c\right) \cdot\left(g_{2,1}\left(x, E_{1}(F(t)) ; E_{2}(F(t))\right)-\omega_{0}(t)\right)+i E_{3}(F(t))\right], \\
u_{1,2}(x, t):=g_{1,2}\left(x, E_{1}(F(t)) ; E_{2}(F(t))\right) \\
\cdot \exp \left[i\left(3 E_{2}(F(t))+B_{1}\left(E_{2}(F(t))\right)-c\right) \cdot\left(g_{2,2}\left(x, E_{1}(F(t)) ; E_{2}(F(t))\right)-\omega_{0}(t)\right)+i E_{3}(F(t))\right]
\end{gathered}
$$

are solutions of the Equation (13); the expression $E_{2}(F(t))$ must verify the condition $E_{2}(F(t)) \in I$.

Now, for $a \in I$, we choose $k=3 a+B_{2}(a)-c$. From the equality (33) it follows $k^{2}=\left(a+B_{2}(a)\right) a^{2}$. We remark that the equation (11) becomes in this case the Equation (20), with $B=B_{2}(a)>0$. Assumptions of Lemma $\left.4, i i\right)$, are satisfied.

Let $g_{1,3}\left(x, c_{1} ; a\right)$ respectively $g_{2,3}\left(x, c_{1} ; a\right)$, the functions defined by the Formula (25), respectively (26), with $B=B_{2}(a)$.

According to Lemma 4, the function $g_{1,3}\left(\cdot, c_{1} ; a\right)$ is a solution of the Equation (11) and $g_{2,3}^{\prime}=\frac{1}{g_{1,3}^{2}}$. Let us consider the function

$$
\varphi_{2,1}\left(x, t, c_{1} ; a\right):=g_{1,3}\left(x, c_{1} ; a\right) e^{i\left(3 a+B_{2}(a)-c\right) g_{2,3}\left(x, c_{1} ; a\right)} v\left(t ; 3 a+B_{2}(a)-c\right) .
$$

From Proposition 8 it follows that the function $\varphi_{2,1}\left(\cdot, \cdot, c_{1} ; a\right)$ is a solution of the Equation (13), which depends on $c_{1}$ and $a$. Proposition 4 implies that the function

$$
\begin{gathered}
u_{2,1}(x, t):=g_{1,3}\left(x, E_{1}(F(t)) ; E_{2}(F(t))\right) \\
\cdot \exp \left[i\left(3 E_{2}(F(t))+B_{2}\left(E_{2}(F(t))\right)-c\right) \cdot\left(g_{2,3}\left(x, E_{1}(F(t)) ; E_{2}(F(t))\right)-\omega_{0}(t)\right)+i E_{3}(F(t))\right]
\end{gathered}
$$

is a solution of the Equation (13); the composition $E_{2}(F(t))$ must verify the condition $E_{2}(F(t)) \in I$.

For the last example we do not ask anymore (necessarily) $a \in I$.

Let $a$ be a real root of the equation $a^{3}=(3 a-c)^{2}$. There is at least one such solution for that the equation considered has degree three. From $a^{3}=(3 a-c)^{2}$ it follows $a \geq 0$ and if $a=0$, then $c=0$. Hence for $c \neq 0$, it follows $a>0$. For $c=0$, we shall take the root $a=9>0$.

We select $k=3 a-c$. From the equality $a^{3}=(3 a-c)^{2}$ we find $k^{2}=a^{3}$. One remark that the Equation (11) becomes in this case the Equation (20), with $B=0$. Assumptions of Lemma 4 , (iii), are satisfied.

Let $g_{1,4}\left(x, c_{1}\right)$ respectively $g_{2,4}\left(x, c_{1}\right)$ be the functions defined by the Formula (27), respectively (28).

According to Lemma 4, the function $g_{1,4}\left(\cdot, c_{1}\right)$ is a solution of the Equation (11) and $g_{2,4}^{\prime}=\frac{1}{g_{1,4}^{2}}$. From Proposition 8 it follows that the function $\varphi_{3,1}\left(\cdot, \cdot, c_{1}\right)$ given by

$$
\varphi_{3,1}\left(x, t, c_{1}\right):=g_{1,4}\left(x, c_{1}\right) e^{i(3 a-c) g_{2,4}\left(x, c_{1}\right)} v(t ; 3 a-c) .
$$


is a solution of the Equation (13), which depends on $c_{1}$. Proposition 4 implies that the function

$$
u_{3,1}(x, t):=g_{1,4}\left(x, E_{1}(F(t))\right) e^{i(3 a-c) \cdot\left(g_{2,4}\left(x, E_{1}(F(t))\right)-\omega_{0}(t)\right)+i E_{2}(F(t))}
$$

is a solution of the Equation (13); where $a$ is a real root of the equation $a^{3}=(3 a-c)^{2}$ if $c \neq 0$; and if $c=0$, then $a=9$.

Remark 5. From the above solutions of the Equation (13) one obtains solutions corresponding to the PDE

$$
i h^{\alpha}(t) \frac{\partial u}{\partial t^{\alpha}}+\frac{\partial^{2} u}{\partial x^{2}}+\left(c-b|u|^{2}\right) u=0, \quad(b>0)
$$

using Remark 1, (c), with $\rho=\frac{\sqrt{2}}{\sqrt{b}}$.

\section{Solutions of Multitime NLSE Whose Argument Is Independent of $x$}

Let $I_{1}, I_{2}$ be two intervals, $I_{1} \subseteq \mathbb{R}, I_{2} \subseteq[0, \infty)$. Let $D$ be an open subset of $\mathbb{R}^{m}$. Let us consider the $\mathcal{C}^{1}$ functions $f: I_{1} \times I_{2} \rightarrow \mathbb{R}, h^{\alpha}: D \rightarrow \mathbb{R}, \alpha \in\{1,2, \ldots, m\}$, which define the multitime nonlinear Schrödinger PDE (2).

In this Section, we shall determine exact solutions of multitime NLSE (2) of exponential form $u(x, t)=\varphi(x, t) e^{i \omega(t)}$, with $\varphi(x, t) \in \mathbb{R}, \omega(t) \in \mathbb{R}$, i.e., the argument $\omega$ of $u$ does not depend on $x$. The solutions of this form are suggested by the solutions in the previous sections and by the question: Are there other solutions of this type? These solutions are in fact extensions of spatial solitons in the paper [16].

Proposition 9. Let $\varphi: I_{0} \times D_{0} \rightarrow \mathbb{R}, \omega: D_{0} \rightarrow \mathbb{R}$; suppose that $\omega$ is a $\mathcal{C}^{1}$ function. Then the function

$$
u: I_{0} \times D_{0} \rightarrow \mathbb{C}, \quad u(x, t)=\varphi(x, t) e^{i \omega(t)}, \quad \text { for all }(x, t) \in I_{0} \times D_{0}
$$

is a solution of multitime NLSE (2) if and only if $\varphi$ is of class $\mathcal{C}^{1}$, there exists $\frac{\partial^{2} \varphi}{\partial x^{2}}$, and for any $(x, t) \in I_{0} \times D_{0}$, the following three conditions are satisfied:

$$
\begin{gathered}
(\varphi(x, t))^{2} \in I_{2}, \\
h^{\alpha}(t) \frac{\partial \varphi}{\partial t^{\alpha}}(x, t)=0, \\
h^{\alpha}(t) \frac{\partial \omega}{\partial t^{\alpha}}(t) \cdot \varphi(x, t)-\frac{\partial^{2} \varphi}{\partial x^{2}}(x, t)-f\left(x,(\varphi(x, t))^{2}\right) \varphi(x, t)=0 .
\end{gathered}
$$

Proof. We remark that $|u|^{2} \in I_{2}$ if and only if $\varphi^{2} \in I_{2}$.

The function $\omega$ is of class $\mathcal{C}^{1}$.

From the equalities $u(x, t)=\varphi(x, t) e^{i \omega(t)}, \varphi(x, t)=u(x, t) e^{-i \omega(t)}$, it follows that the function $u$ is of class $\mathcal{C}^{1}$ and there exists $\frac{\partial^{2} u}{\partial x^{2}}$ if and only if $\varphi$ is of class $\mathcal{C}^{1}$ and there exists $\frac{\partial^{2} \varphi}{\partial x^{2}}$.

The function $u$ verifies the relation (2) if and only if, for any $(x, t) \in I_{0} \times D_{0}$, we have

$$
i h^{\alpha} \frac{\partial \varphi}{\partial t^{\alpha}} \cdot e^{i \omega(t)}-h^{\alpha} \frac{\partial \omega}{\partial t^{\alpha}}(t) \cdot \varphi \cdot e^{i \omega(t)}+\frac{\partial^{2} \varphi}{\partial x^{2}} \cdot e^{i \omega(t)}+f\left(x, \varphi^{2}\right) \varphi \cdot e^{i \omega(t)}=0,
$$

which is equivalent to

$$
i h^{\alpha} \frac{\partial \varphi}{\partial t^{\alpha}}-h^{\alpha} \frac{\partial \omega}{\partial t^{\alpha}}(t) \cdot \varphi+\frac{\partial^{2} \varphi}{\partial x^{2}}+f\left(x, \varphi^{2}\right) \varphi=0
$$


and the above equality is valid if and only if the equalities (38) and (39) are satisfied.

\subsection{Preresiquites: First Integrals}

Let $t_{0} \in D$, for which there is at least one index $\alpha_{0} \in\{1, \ldots, m\}$, such that $h^{\alpha_{0}}\left(t_{0}\right) \neq 0$.

Let $\beta_{1}, \beta_{2}, \ldots, \beta_{m-1}$, be distinct elements of the set $\{1,2, \ldots, m\} \backslash\left\{\alpha_{0}\right\}$, written in ascending order.

Consider the $\mathcal{C}^{1}$ first integrals $F_{1}, F_{2}, \ldots, F_{m-1}: V \rightarrow \mathbb{R}$ of ODEs system (3), with $V \subseteq D, t_{0} \in V, V$ open and connected, such that $h^{\alpha_{0}}(t) \neq 0$, for any $t \in V$, and

$$
\operatorname{det}\left(\frac{\partial F_{\alpha}}{\partial t^{\beta_{j}}}(t)\right)_{1 \leq \alpha, j \leq m-1} \neq 0, \quad \text { for all } t \in V
$$

(There exist the functions $F_{1}, F_{2}, \ldots, F_{m-1}$ with above properties).

We denote by $F(\cdot)$ the vector field $F=\left(F_{1}, F_{2}, \ldots, F_{m-1}\right): V \rightarrow \mathbb{R}^{m-1}$.

Renumbering the indices, we can assume that $\alpha_{0}=m$. Hence we have $h^{m}(t) \neq 0$, for any $t \in V$, and

$$
\operatorname{det}\left(\frac{\partial F_{\alpha}}{\partial t^{\beta}}(t)\right)_{1 \leq \alpha, \beta \leq m-1} \neq 0, \quad \text { for all } t \in V \text {. }
$$

Let $F_{m}(t)=t^{m}$. Denote by $H(\cdot)$ the vector field $H=\left(F_{1}, \ldots, F_{m-1}, F_{m}\right): V \rightarrow \mathbb{R}^{m}$, i.e., $H=\left(F, F_{m}\right)$. We can easily see that the Jacobian of $H$ is equal to the determinant in relation (41), hence it is nonzero on $V$. From the inverse function theorem, it follows that there are two open sets, $V_{0} \subseteq V, V_{1} \subseteq \mathbb{R}^{m}$, with $t_{0} \in V_{0}$, such that the function (a restriction of $H$ ) $\left.H\right|_{V_{0}}: V_{0} \rightarrow V_{1}$ is a $\mathcal{C}^{1}$ is diffeomorphism. Let $G=\left(G_{1}, \ldots, G_{m}\right): V_{1} \rightarrow V_{0}$ be the inverse of this diffeomorphism.

Notation: For $s=\left(s^{1}, \ldots, s^{m-1}, s^{m}\right)$, we denote $\widetilde{s}:=\left(s^{1}, \ldots, s^{m-1}\right)$; hence we have $s=\left(\widetilde{s}, s^{m}\right)$. The following equalities:

$$
G(H(t))=t \text {, for all } t \in V_{0} ; \quad H(G(s))=s \text {, for all } s \in V_{1} \text {. }
$$

are satisfied. The second above equality is $\left(F\left(G(s), G_{m}(s)\right)=\left(\widetilde{s}, s^{m}\right)\right.$, hence it results

$$
\begin{gathered}
F(G(s))=F\left(G\left(\widetilde{s}, s^{m}\right)\right)=\widetilde{s}, \quad \text { for all } s=\left(\widetilde{s}, s^{m}\right) \in V_{1} ; \\
G_{m}(s)=G_{m}\left(\widetilde{s}, s^{m}\right)=s^{m}, \quad \text { for all } s=\left(\widetilde{s}, s^{m}\right) \in V_{1} .
\end{gathered}
$$

We need the vector field $h(t):=\left(h^{1}(t), h^{2}(t), \ldots, h^{m}(t)\right), t \in D$.

For $t \in V$, we denote $S_{t}:=\operatorname{Sp}\left\{\nabla F_{1}(t), \nabla F_{2}(t), \ldots, \nabla F_{m-1}(t)\right\} \subseteq \mathbb{R}^{m}$. From the relation (41) it follows that $\operatorname{dim} S_{t}=m-1$. Hence $\operatorname{dim} S_{t}^{\perp}=1$.

For any $\beta \in\{1,2, \ldots, m-1\}$, we have $\left\langle h(t), \nabla F_{\beta}(t)\right\rangle=0$, since the functions $F_{\beta}$ are first integrals of the ODEs system (3); hence $h(t) \in S_{t}^{\perp}$. Since $h(t) \neq 0$, it follows that $\{h(t)\}$ is a basis of the vector space $S_{t}^{\perp}$ (for all $t \in V$ ).

Lemma 5. Suppose that the above conditions are satisfied. Then

(a) For any $s \in V_{1}$, the relation $h^{m}(G(s)) \frac{\partial G}{\partial s^{m}}(s)=h(G(s))$ is satisfied.

(b) Let $D_{0} \subseteq D$, be an open neighborhood of $t_{0}$. Whatever the $\mathcal{C}^{1}$ function $w: D_{0} \rightarrow \mathbb{R}$, we have

$$
h^{\alpha}(G(s)) \frac{\partial w}{\partial t^{\alpha}}(G(s))=h^{m}(G(s)) \frac{\partial}{\partial s^{m}}(w(G(s))), \quad \text { for all } s \in H\left(D_{0} \cap V_{0}\right) .
$$

Proof. (a) Since $H \circ G(s)=s$, it follows that for any $s \in V_{1}$ and for any $\beta \in\{1,2, \ldots, m-1\}$, we have $F_{\beta}(G(s))=s^{\beta}$. Taking the derivative with respect to $s^{m}$, we obtain

$$
\left\langle\nabla F_{\beta}(G(s)), \frac{\partial G}{\partial s^{m}}(s)\right\rangle=0, \quad \text { for all } s \in V_{1}, \text { for all } \beta \in\{1,2, \ldots, m-1\} \text {. }
$$


Hence $\forall s \in V_{1}, \frac{\partial G}{\partial s^{m}}(s) \in S_{G(s)}^{\perp}=S p\{h(G(s))\}$. There exists $\lambda(s) \in \mathbb{R}$ such that $\frac{\partial G}{\partial s^{m}}(s)=\lambda(s) h(G(s))$. Identifying the components on the position $m$, we find $\frac{\partial G_{m}}{\partial s^{m}}(s)=\lambda(s) h^{m}(G(s))$. Since $G_{m}(s)=s^{m}$, it follows $\lambda(s) h^{m}(G(s))=1$.

We remark that if we multiply by $h^{m}(G(s))$, the equality $\frac{\partial G}{\partial s^{m}}(s)=\lambda(s) h(G(s))$, becomes the equality from the point $a$ ).

(b) If $s \in H\left(D_{0} \cap V_{0}\right)$, then $G(s) \in G \circ H\left(D_{0} \cap V_{0}\right)=D_{0} \cap V_{0} \subseteq D_{0}$.

For any $s \in H\left(D_{0} \cap V_{0}\right)$, we have

$$
\begin{gathered}
h^{m}(G(s)) \frac{\partial}{\partial s^{m}}(w(G(s)))=h^{m}(G(s))\left\langle\nabla w(G(s)), \frac{\partial G}{\partial s^{m}}(s)\right\rangle= \\
=\left\langle\nabla w(G(s)), h^{m}(G(s)) \frac{\partial G}{\partial s^{m}}(s)\right\rangle=\langle\nabla w(G(s)), h(G(s))\rangle= \\
=h^{\alpha}(G(s)) \frac{\partial w}{\partial t^{\alpha}}(G(s)),
\end{gathered}
$$

and the statement is proved.

Remark 6. Let $D_{0} \subseteq D$, be an open neighborhood of $t_{0}$ and let $s_{0}:=H\left(t_{0}\right)$. Let $J_{1}, J_{2}, \ldots, J_{m}$ be open intervals, such that $s_{0}^{\alpha} \in J_{\alpha}$, for all $\alpha \in\{1,2, \ldots, m\}$, and $W_{1}:=J_{1} \times J_{2} \times \ldots \times J_{m} \subseteq H\left(D_{0} \cap V_{0}\right) \subseteq V_{1}$ (there exist such intervals since the set $H\left(D_{0} \cap V_{0}\right)$ is open and $s_{0}=H\left(t_{0}\right) \in H\left(D_{0} \cap V_{0}\right)$ ).

Because $s_{0}^{m}=t_{0}^{m}$, we have $t_{0}^{m} \in J_{m}$.

The set $W_{0}:=G\left(W_{1}\right)$ is open since $W_{1}$ is open and $G$ is a diffeomorphism.

Since $s_{0} \in W_{1}$, we deduce $t_{0}=G\left(s_{0}\right) \in G\left(W_{1}\right)=W_{0}$.

From $W_{1} \subseteq H\left(D_{0} \cap V_{0}\right)$, it follows that $W_{0}=G\left(W_{1}\right) \subseteq G \circ H\left(D_{0} \cap V_{0}\right)=D_{0} \cap V_{0}$.

Hence, the set $W_{0}=G\left(W_{1}\right)$ is an open neighborhood of $t_{0}$ and $W_{0} \subseteq D_{0} \cap V_{0}$.

We have $H\left(W_{0}\right)=H \circ G\left(W_{1}\right)=W_{1}$, i.e., $H\left(W_{0}\right)=W_{1}$. It follows that for any $t \in W_{0}$, we can write $H(t)=\left(F(t), t^{m}\right) \in W_{1}$, whence we deduce

$$
\begin{gathered}
F(t) \in J_{1} \times J_{2} \times \ldots \times J_{m-1}, \quad \text { for all } t \in W_{0} . \\
\left(F(t), s^{m}\right) \in J_{1} \times J_{2} \times \ldots \times J_{m}, \quad \text { for all } t \in W_{0}, \text { for all } s^{m} \in J_{m} .
\end{gathered}
$$

Proposition 10. Let $D_{0} \subseteq D$ be an open neighborhood of $t_{0}$. Denote by $q: D_{0} \rightarrow \mathbb{R}$ an arbitrary function. We select the sets $W_{1}, W_{0}$ as in the Remark 6.

(a) If the $\mathcal{C}^{1}$ function $w: D_{0} \rightarrow \mathbb{R}$ verifies, for all $t \in D_{0}$, the equation

$$
h^{\alpha}(t) \frac{\partial w}{\partial t^{\alpha}}(t)=q(t)
$$

then

$$
\begin{gathered}
w(G(s))=w\left(G\left(\widetilde{s}, t_{0}^{m}\right)\right)+\int_{t_{0}^{m}}^{s^{m}} \frac{q(G(\widetilde{s}, \tau))}{h^{m}(G(\widetilde{s}, \tau))} \mathrm{d} \tau, \quad \text { for all } s=\left(\widetilde{s}, s^{m}\right) \in W_{1}, \\
w(t)=w\left(G\left(F(t), t_{0}^{m}\right)\right)+\int_{t_{0}^{m}}^{t^{m}} \frac{q(G(F(t), \tau))}{h^{m}(G(F(t), \tau))} \mathrm{d} \tau, \quad \text { for all } t \in W_{0} .
\end{gathered}
$$


(b) Suppose that the function $q$ is of class $\mathcal{C}^{1}$. Let $W$ be an open neighborhood of $t_{0}, W \subseteq V$ (as example $W=W_{0}$ ). Let us consider a $\mathcal{C}^{1}$ function $E: U \rightarrow \mathbb{R}, U \subseteq \mathbb{R}^{m-1}, U$ open. Suppose that for any $t \in W$ we have $F(t) \in U$. Then, the function $w: W \cap W_{0} \rightarrow \mathbb{R}$, defined by

$$
w(t)=E(F(t))+\int_{t_{0}^{m}}^{t^{m}} \frac{q(G(F(t), \tau))}{h^{m}(G(F(t), \tau))} \mathrm{d} \tau, \quad \text { for all } t \in W \cap W_{0},
$$

is of class $\mathcal{C}^{1}$, and verifies the relation (42), for all $t \in W \cap W_{0}$.

Proof. (a) Since $w$ is of class $\mathcal{C}^{1}$, from the relation (42) we deduce that the function $q$ is continuous.

We fix $\widetilde{s} \in J_{1} \times J_{2} \times \ldots \times J_{m-1}$. Hence for any $s^{m} \in J_{m}$, we have $\left(\widetilde{s}, s^{m}\right) \in W_{1} \subseteq H\left(D_{0} \cap V_{0}\right)$. We can define the function

$$
\lambda: J_{m} \rightarrow \mathbb{R}, \quad \lambda(\tau)=w(G(\widetilde{s}, \tau)), \quad \text { for all } \tau \in J_{m} .
$$

From Lemma 5, (b), and the equality (42), it follows

$$
q(G(\widetilde{s}, \tau))=h^{\alpha}(G(\widetilde{s}, \tau)) \frac{\partial w}{\partial t^{\alpha}}(G(\widetilde{s}, \tau))=h^{m}(G(\widetilde{s}, \tau)) \frac{\mathrm{d} \lambda}{\mathrm{d} \tau}(\tau), \quad \text { for all } \tau \in J_{m}
$$

i.e, $\frac{\mathrm{d} \lambda}{\mathrm{d} \tau}(\tau)=\frac{q(G(\widetilde{s}, \tau))}{h^{m}(G(\widetilde{s}, \tau))}$, hence we obtain

$$
\lambda\left(s^{m}\right)-\lambda\left(t_{0}^{m}\right)=\int_{t_{0}^{m}}^{s^{m}} \frac{q(G(\widetilde{s}, \tau))}{h^{m}(G(\widetilde{s}, \tau))} \mathrm{d} \tau, \quad \text { for all } s^{m} \in J_{m} .
$$

We find that the above relationship coincides with the relationship (43).

Let $t \in W_{0}$. According to Remark 6, we have $H(t)=\left(F(t), t^{m}\right) \in W_{1}$.

In the Formula (43), we take $s=H(t)=\left(F(t), t^{m}\right)$, i.e., $\widetilde{s}=F(t), s^{m}=t^{m}$, and we obtain the Formula (44) (since $G(H(t))=t$ ).

(b) Since $q$ is of class $\mathcal{C}^{1}$, it follows that $w$ is of class $\mathcal{C}^{1}$.

The variables of the function $E$ are denoted by $\left(s^{1}, s^{2}, \ldots, s^{m-1}\right)$.

Let $E_{1}: W_{1} \rightarrow \mathbb{R}, \quad E_{1}(s)=\frac{q(G(s))}{h^{m}(G(s))}, \quad$ for all $s \in W_{1}$. Then

$h^{\alpha}(t) \frac{\partial w}{\partial t^{\alpha}}(t)=h^{\alpha}(t) \sum_{j=1}^{m-1} \frac{\partial E}{\partial s^{j}}(F(t)) \cdot \frac{\partial F_{j}}{\partial t^{\alpha}}(t)+$

$+h^{\alpha}(t) \int_{t_{0}^{m}}^{t^{m}} \sum_{j=1}^{m-1} \frac{\partial E_{1}}{\partial s^{j}}((F(t), \tau)) \cdot \frac{\partial F_{j}}{\partial t^{\alpha}}(t) \mathrm{d} \tau+h^{m}(t) \cdot \frac{q\left(G\left(F(t), t^{m}\right)\right)}{h^{m}\left(G\left(F(t), t^{m}\right)\right)}=$

$=\sum_{j=1}^{m-1} \frac{\partial E}{\partial s^{j}}(F(t)) \cdot h^{\alpha}(t) \frac{\partial F_{j}}{\partial t^{\alpha}}(t)+\int_{t_{0}^{m}}^{t^{m}} \sum_{j=1}^{m-1} \frac{\partial E_{1}}{\partial s^{j}}((F(t), \tau)) \cdot h^{\alpha}(t) \frac{\partial F_{j}}{\partial t^{\alpha}}(t) \mathrm{d} \tau$

$+h^{m}(t) \cdot \frac{q(G(H(t)))}{h^{m}(G(H(t)))}=0+0+h^{m}(t) \cdot \frac{q(t)}{h^{m}(t)}=q(t)$.

Proposition 11. Let $t_{0} \in D$. Let us consider the $\mathcal{C}^{1}$ functions $F_{1}, F_{2}, \ldots, F_{m-1}: V \rightarrow \mathbb{R}$ as first integrals for the ODEs system (3), with $V \subseteq D, t_{0} \in V, V$ open and connected. Let $F(t):=\left(F_{1}(t), F_{2}(t), \ldots, F_{m-1}(t)\right), t \in V$.

(a) Suppose there exists $\alpha_{0} \in\{1,2, \ldots, m\}$, such that for all $t \in V$, we have $h^{\alpha_{0}}(t) \neq 0$, and the condition (40) is satisfied.

Let $\Psi: D_{0} \rightarrow \mathbb{R}$ be a $\mathcal{C}^{1}$ first integral of the ODEs system (3), with $t_{0} \in D_{0} \subseteq D, D_{0}$ open. 
Then, there exists an open neighborhood $W_{0}$ of $t_{0}$, with $W_{0} \subseteq D_{0} \cap V$; there exists $U_{0} \subseteq \mathbb{R}^{m-1}$, $U_{0}$ open, and there exists a $\mathcal{C}^{1}$ function $E_{0}: U_{0} \rightarrow \mathbb{R}$ such that

$$
F(t) \in U_{0} \text { and } \Psi(t)=E_{0}(F(t)), \text { for all } t \in W_{0} .
$$

(b) Let $U \subseteq \mathbb{R}^{m-1}, U$ open, and let $E: U \rightarrow \mathbb{R}$ be a function of class $\mathcal{C}^{1}$. Let $W \subseteq V$, $W$ open, such that, for any $t \in W$ we have $F(t) \in U$.

Then, the function $\Psi: W \rightarrow \mathbb{R}, \Psi(t)=E(F(t))$, for all $t \in W$, is a first integral for the ODEs system (3).

Proof. (a) Renumbering the indices, we can assume that $\alpha_{0}=m$.

We choose $W_{1}$ and $W_{0}$ as in the Remark 6. Let $U_{0}:=J_{1} \times J_{2} \times \ldots \times J_{m-1}$; obviously, $U_{0}$ is open.

According to Remark 6, the set $W_{0}$ is open, $t_{0} \in W_{0}, W_{0} \subseteq D_{0} \cap V_{0} \subseteq D_{0} \cap V$, and $F(t) \in U_{0}$, for all $t \in W_{0}$.

Applying Proposition 10, (a), for $w=\Psi, q=0$, it follows that, for all $t \in W_{0}$, we have $\Psi(t)=\Psi\left(G\left(F(t), t_{0}^{m}\right)\right)$.

It is sufficient to choose $E_{0}: U_{0} \rightarrow \mathbb{R}, E_{0}(\widetilde{s})=\Psi\left(G\left(\widetilde{s}, t_{0}^{m}\right)\right)$, for all $\widetilde{s} \in U_{0}$ $\left(\left(\widetilde{s}, t_{0}^{m}\right) \in U_{0} \times J_{m}=W_{1} \subseteq V_{1} ; G\left(W_{1}\right)=W_{0} \subseteq D_{0}\right)$; obviously, the function $E$ is of class $\mathcal{C}^{1}$.

(b) A calculation similar to the proof of Proposition 10, (b) is made.

\subsection{The Theorem about the Solutions with the Argument Independent of $x$}

Let us insist again on solutions of exponential form of the multitime NLSE (2).

Proposition 12. Let $\left(x_{0}, t_{0}\right) \in I_{1} \times D$. Suppose there exists $\alpha_{0} \in\{1,2, \ldots, m\}$, such that $h^{\alpha_{0}}\left(t_{0}\right) \neq 0$.

We need the functions $\varphi: L \times D_{1} \rightarrow \mathbb{R}, \omega: D_{1} \rightarrow \mathbb{R}$, $\omega$ of class $\mathcal{C}^{1}$ ( $L$ subinterval of $I_{1}$, with $x_{0} \in L ; D_{1} \subseteq D, D_{1}$ open, with $t_{0} \in D_{1}$ ). Suppose that the function

$$
u: I_{0} \times D_{0} \rightarrow \mathbb{C}, \quad u(x, t)=\varphi(x, t) e^{i \omega(t)}, \quad \text { for all }(x, t) \in L \times D_{1},
$$

is a solution for the multitime NLSE (2). We add the condition $\varphi\left(x_{0}, t_{0}\right)>0$.

Let us consider the $\mathcal{C}^{1}$ functions $F_{1}, F_{2}, \ldots, F_{m-1}: V \rightarrow \mathbb{R}$ as first integrals of the ODEs system (3), with $V \subseteq D, t_{0} \in V, V$ open and connected, such that for all $t \in V$, we have $h^{\alpha_{0}}(t) \neq 0$, and the condition (40) is satisfied.

We need the vector field $F(t):=\left(F_{1}(t), F_{2}(t), \ldots, F_{m-1}(t)\right), t \in V$.

Then

- there exists $I_{0}$ subinterval of $L$, with $x_{0} \in I_{0}$ (if $L$ is an open interval, then $I_{0}$ is open also);

- there exists $W_{0}$ an open neighborhood of $t_{0}, W_{0} \subseteq V \cap D_{1}$;

- there exists $U_{0} \subseteq \mathbb{R}^{m-1}, U_{0}$ open; such that

- there exist the $\mathcal{C}^{1}$ functions $E_{1}, E_{2}, E_{3}, E_{4}: U_{0} \rightarrow \mathbb{R}, \omega_{0}: W_{0} \rightarrow \mathbb{R}$,

(i) $\varphi(x, t)>0$, for all $(x, t) \in I_{0} \times W_{0}$;

(ii) $F(t) \in U_{0}$, for all $t \in W_{0}$;

(iii) $E_{1}(\widetilde{s})>0$, for all $\widetilde{s} \in U_{0}$; and $\left(E_{1}(\widetilde{s})\right)^{2} \in I_{2}$, for all $\widetilde{s} \in U_{0}$;

(iv) $\varphi\left(x_{0}, t\right)=E_{1}(F(t))$, for all $t \in W_{0}$;

(v) $\frac{\partial \varphi}{\partial x}\left(x_{0}, t\right)=E_{2}(F(t))$, for all $t \in W_{0}$;

(vi) $\omega(t)=-\omega_{0}(t) E_{3}(F(t))+E_{4}(F(t))$, for all $t \in W_{0}$;

(vii) $h^{\alpha}(t) \frac{\partial \omega_{0}}{\partial t^{\alpha}}(t)=1$, for all $t \in W_{0}$.

Proof. Renumbering the indices, we can suppose that $\alpha_{0}=m$. 
Since $\varphi\left(x_{0}, t_{0}\right)>0$, there exists $D_{0} \subseteq D_{1}, D_{0}$ an open neighborhood of $t_{0}$, and there exists $I_{0}$ subinterval of $L$, with $x_{0} \in I_{0}$, such that $\varphi(x, t)>0$, for all $(x, t) \in I_{0} \times D_{0}$ (if $L$ is an open interval, then $I_{0}$ can be selected as open interval).

We select $W_{1}$ and $W_{0}$ as in Remark 6, i.e., $J_{1}, J_{2}, \ldots, J_{m}$ are open intervals, such that $s_{0}^{\alpha} \in J_{\alpha}$ (whatever the index $\left.\alpha\right), s_{0}=H\left(t_{0}\right)$, and

$W_{1}:=J_{1} \times J_{2} \times \ldots \times J_{m} \subseteq H\left(D_{0} \cap V_{0}\right), W_{0}:=G\left(W_{1}\right)$.

We choose $U_{0}:=J_{1} \times J_{2} \times \ldots \times J_{m-1}$; obvious that $U_{0}$ is open.

According to Remark 6, the set $W_{0}$ is open, $t_{0} \in W_{0}, W_{0} \subseteq D_{0} \cap V_{0} \subseteq D_{1} \cap V$, and $F(t) \in U_{0}$, for all $t \in W_{0}$.

Since $W_{0} \subseteq D_{0}$, we deduce that, for all $(x, t) \in I_{0} \times W_{0}$, we have $\varphi(x, t)>0$.

Let $E: U_{0} \rightarrow W_{0}, E(\widetilde{s})=G\left(\widetilde{s}, t_{0}^{m}\right)$, for all $\widetilde{s} \in U_{0}\left(\left(\widetilde{s}, t_{0}^{m}\right) \in U_{0} \times J_{m}=W_{1}\right)$; obviously that $E$ is a $\mathcal{C}^{1}$ function.

According to Proposition 9, the function $\varphi(x, \cdot)$ verifies the relation (38) on the set $D_{0}$. Proposition 10, $(a)$, (with $w(\cdot)=\varphi(x, \cdot)$ and $q=0)$, implies

$\varphi(x, t)=\varphi(x, E(F(t)))$, for all $t \in W_{0}$, for all $x \in I_{0}$; taking the derivative with respect to $x$, we obtain $\frac{\partial \varphi}{\partial x}(x, t)=\frac{\partial \varphi}{\partial x}(x, E(F(t)))$, for all $t \in W_{0}$, for all $x \in I_{0}$.

Let $E_{1}, E_{2}: U_{0} \rightarrow \mathbb{R}$ be defined by

$E_{1}(\widetilde{s})=\varphi\left(x_{0}, E(\widetilde{s})\right), \quad E_{2}(\widetilde{s})=\frac{\partial \varphi}{\partial x}\left(x_{0}, E(\widetilde{s})\right), \quad$ for all $\widetilde{s} \in U_{0}$.

For all $t \in W_{0}$, we have $E_{1}(F(t))=\varphi\left(x_{0}, E(F(t))=\varphi\left(x_{0}, t\right)\right.$ and $E_{2}(F(t))=\frac{\partial \varphi}{\partial x}\left(x_{0}, E(F(t))\right)=\frac{\partial \varphi}{\partial x}\left(x_{0}, t\right)$; we proved the statements $\left.i v\right)$ and $\left.v\right)$.

For any $\widetilde{s} \in U_{0}$, we have $E(\widetilde{s}) \in W_{0} \subseteq D_{0}$; it follows that $\varphi\left(x_{0}, E(\widetilde{s})\right)>0$, i.e., $E_{1}(\widetilde{s})>0$.

From Proposition 9, we deduce that, for any $t \in W_{0}$, we have $\left(\varphi\left(x_{0}, t\right)\right)^{2} \in I_{2}$.

For any $\widetilde{s} \in U_{0}$, we have $E(\widetilde{s}) \in W_{0}$; it follows that $\varphi\left(x_{0}, E(\widetilde{s})\right)^{2} \in I_{2}$, i.e., $\left(E_{1}(\widetilde{s})\right)^{2} \in I_{2}$. So we also proved the statements from iii).

From $\frac{\partial \varphi}{\partial x}(x, t)=\frac{\partial \varphi}{\partial x}(x, E(F(t)))$, it follows $\frac{\partial^{2} \varphi}{\partial x^{2}}(x, t)=\frac{\partial^{2} \varphi}{\partial x^{2}}(x, E(F(t)))$, for all $t \in W_{0}$, for all $x \in I_{0}$.

If in the relation (39) it counts $x=x_{0}$, then for any $t \in W_{0}$ one obtains

$$
h^{\alpha}(t) \frac{\partial \omega}{\partial t^{\alpha}}(t)=\frac{1}{E_{1}(F(t))} \cdot \frac{\partial^{2} \varphi}{\partial x^{2}}\left(x_{0}, E(F(t))\right)+f\left(x_{0},\left(E_{1}(F(t))\right)^{2}\right) .
$$

We define the function $E_{3}: U_{0} \rightarrow \mathbb{R}$,

$E_{3}(\widetilde{s})=-\left(\frac{1}{E_{1}(\widetilde{s})} \cdot \frac{\partial^{2} \varphi}{\partial x^{2}}\left(x_{0}, E(\widetilde{s})\right)+f\left(x_{0},\left(E_{1}(\widetilde{s})\right)^{2}\right)\right), \quad$ for all $\widetilde{s} \in U_{0}$.

From the previous formula it follows that $E_{3}$ is a continuous function. However, it does not turn out that $E_{3}$ is of class $\mathcal{C}^{1}$. This remains to be proved.

The equality (46) becomes $h^{\alpha}(t) \frac{\partial \omega}{\partial t^{\alpha}}(t)=-E_{3}(F(t))$, for all $t \in W_{0}$.

We apply Proposition 10, $(a)$, for $w=\omega$ and $q(t)=-E_{3}(F(t))$; using the equality $F(G(\widetilde{s}, \tau))=\widetilde{s}$, we obtain

$$
\omega(G(s))=\omega\left(G\left(\widetilde{s}, t_{0}^{m}\right)\right)-\int_{t_{0}^{m}}^{s^{m}} \frac{E_{3}(\widetilde{s})}{h^{m}(G(\widetilde{s}, \tau))} \mathrm{d} \tau, \quad \forall \widetilde{s} \in U_{0}, \forall s^{m} \in J_{m} .
$$

We fix $p \in J_{m}, p \neq t_{0}^{m}$. Let $E_{5}(\widetilde{s}):=\int_{t_{0}^{m}}^{p} \frac{1}{h^{m}(G(\widetilde{s}, \tau))} \mathrm{d} \tau$, for all $\widetilde{s} \in U_{0}$.

Since for any $t \in V$ we have $h^{m}(t) \neq 0$, we deduce that $h^{m}$ has constant sign on $V$; it follows that, for all $\widetilde{s} \in U_{0}$, we have $E_{5}(\widetilde{s}) \neq 0$. 
From the Formula (47), we obtain $E_{3}(\widetilde{s})=\frac{\omega\left(G\left(\widetilde{s}, t_{0}^{m}\right)\right)-\omega(G(\widetilde{s}, p))}{E_{5}(\widetilde{s})}$, for all $\widetilde{s} \in U_{0}$; from this we deduce that $E_{3}$ is a $\mathcal{C}^{1}$ function.

In Formula (47), we take $s=H(t)=\left(F(t), t^{m}\right)$, i.e., $\widetilde{s}=F(t), s^{m}=t^{m}$, and we obtain

$$
\omega(t)=\omega\left(G\left(F(t), t_{0}^{m}\right)\right)-E_{3}(F(t)) \int_{t_{0}^{m}}^{t^{m}} \frac{1}{h^{m}(G(F(t), \tau))} \mathrm{d} \tau, \quad \text { for all } t \in W_{0} .
$$

We define the functions $E_{4}: U_{0} \rightarrow \mathbb{R}, \omega_{0}: W_{0} \rightarrow \mathbb{R}$ by the formulas $E_{4}(\widetilde{s})=\omega\left(G\left(\widetilde{s}, t_{0}^{m}\right)\right), \quad$ for all $\widetilde{s} \in U_{0} ; \quad \omega_{0}(t)=\int_{t_{0}^{m}}^{t^{m}} \frac{1}{h^{m}(G(F(t), \tau))} \mathrm{d} \tau, \quad$ for all $t \in W_{0}$.

The equality (48) becomes the formula from point $v i$ ).

We apply Proposition 10, (b), for $W=W_{0}, E=0, q=1$; it follows that $\omega_{0}$ verifies the relation of point (vii).

Remark 7. We assume that the hypotheses of Proposition 12 are satisfied, but with $\varphi\left(x_{0}, t_{0}\right)<0$. Setting $\varphi_{1}(x, t):=-\varphi(x, t), \omega_{1}(t):=\pi+\omega(t)$, we have $u(x, t)=\varphi_{1}(x, t) e^{i \omega_{1}(t)}$, and $\varphi_{1}\left(x_{0}, t_{0}\right)=-\varphi\left(x_{0}, t_{0}\right)>0$. The conclusions of Proposition 12 are obtained for the functions $\varphi_{1}$ and $\omega_{1}$, respectively, instead of $\varphi$ and $\omega$, respectively.

Further we shall determine (locally) the exact solutions of multitime NLSE (2) of the form $u(x, t)=\varphi(x, t) e^{i \omega(t)}$, with $\varphi(x, t) \in \mathbb{R}, \omega(t) \in \mathbb{R}$; these solutions being considered in a neighborhood of a point $\left(x_{0}, t_{0}\right)$ for which $u\left(x_{0}, t_{0}\right) \neq 0$ and $\left(h^{1}\left(t_{0}\right), h^{2}\left(t_{0}\right), \ldots, h^{m}\left(t_{0}\right)\right) \neq(0,0, \ldots, 0)$. Proposition 12 presents necessary conditions which must perform (locally) the functions $u\left(x_{0}, \cdot\right), \frac{\partial u}{\partial x}\left(x_{0}, \cdot\right)$ and $\omega$ (taking into account also Remark 7). So we will further assume that these conditions are met.

Suppose that $I_{1}$ is an open interval and that $I_{2}$ has the form $I_{2}:=(\mu, \infty)$, with $\mu \geq 0$. Let $I_{3}:=(\sqrt{\mu}, \infty)$; it is obvious that $I_{3} \subseteq(0, \infty)$.

We consider the ODE (with parameter $k \in \mathbb{R}$ )

$$
y^{\prime \prime}+\left(k+f\left(x, y^{2}\right)\right) y=0 .
$$

For $k \in \mathbb{R}, \lambda \in I_{1}, c_{1} \in I_{3}, c_{2} \in \mathbb{R}$, let $\sigma\left(\cdot, \lambda ; c_{1}, c_{2} ; k\right)$ be the maximal solution $y(\cdot)$ of the Equation (49) which verifies $y(\lambda)=c_{1}$ and $y^{\prime}(\lambda)=c_{2}$. The domain of definition of this solution being the open interval $I\left(\lambda ; c_{1}, c_{2} ; k\right) \subseteq I_{1}$, with $\lambda \in I\left(\lambda ; c_{1}, c_{2} ; k\right)$; the codomain is $I_{3}$.

Let us introduce the set

$M:=\left\{\left(x, \lambda ; c_{1}, c_{2} ; k\right) \mid \lambda \in I_{1}, c_{1} \in I_{3}, c_{2} \in \mathbb{R}, k \in \mathbb{R}, x \in I\left(\lambda ; c_{1}, c_{2} ; k\right)\right\}$.

The set $M$ is open. The function $\sigma: M \rightarrow I_{3}$ is of class $\mathcal{C}^{1}$; the function $\frac{\partial \sigma}{\partial x}: M \rightarrow I_{3}$ is of class $\mathcal{C}^{1}$.

For any $\lambda \in I_{1}, c_{1} \in I_{3}, c_{2} \in \mathbb{R}, k \in \mathbb{R}$, we have

$\sigma\left(\lambda, \lambda ; c_{1}, c_{2} ; k\right)=c_{1} \quad$ and $\quad \frac{\partial \sigma}{\partial x}\left(\lambda, \lambda ; c_{1}, c_{2} ; k\right)=c_{2}$.

Theorem 1. Suppose that $I_{1}$ is an open interval and that there exists $\mu \geq 0$ such that $I_{2}=(\mu, \infty)$; let $I_{3}=(\sqrt{\mu}, \infty)$.

Let $\left(x_{0}, t_{0}\right) \in I_{1} \times D$. Suppose that there exists $\alpha_{0} \in\{1,2, \ldots, m\}$, such that $h^{\alpha_{0}}\left(t_{0}\right) \neq 0$. Let us consider the $\mathcal{C}^{1}$ functions $F_{1}, F_{2}, \ldots, F_{m-1}: V \rightarrow \mathbb{R}$ as first integrals of the ODEs system (3), with $V \subseteq D, t_{0} \in V, V$ open and connected, such that, for all $t \in V$, we have $h^{\alpha_{0}}(t) \neq 0$, and the condition (40) is satisfied.

Denote $F(t):=\left(F_{1}(t), F_{2}(t), \ldots, F_{m-1}(t)\right), t \in V$.

Let $I_{0} \subseteq I_{1}, I_{0}$ interval, with $x_{0} \in I_{0}$; let $W_{0} \subseteq V$, $W_{0}$ open, with $t_{0} \in W_{0}$. 
We need a $\mathcal{C}^{1}$ function $\omega_{0}: W_{0} \rightarrow \mathbb{R}$, such that $h^{\alpha}(t) \frac{\partial \omega_{0}}{\partial t^{\alpha}}(t)=1$, for all $t \in W_{0}$.

Let us consider the $\mathcal{C}^{1}$ functions

$E_{1}: U_{0} \rightarrow I_{3}, E_{2}, E_{3}, E_{4}: U_{0} \rightarrow \mathbb{R}$ (with $U_{0} \subseteq \mathbb{R}^{m-1}, U_{0}$ open); suppose that for any $t \in W_{0}$, we have $F(t) \in U_{0}$.

Let $\omega: W_{0} \rightarrow \mathbb{R}, \omega(t)=-\omega_{0}(t) E_{3}(F(t))+E_{4}(F(t))$, for all $t \in W_{0}$.

(a) Suppose that the function $u: I_{0} \times W_{0} \rightarrow \mathbb{C}$ is a solution of the multitime NLSE (2), of the form $u(x, t)=\varphi(x, t) e^{i \omega(t)}$, for all $(x, t) \in I_{0} \times W_{0}$, with $\varphi(x, t)>0$, for all $(x, t) \in I_{0} \times W_{0}$. If

$$
u\left(x_{0}, t\right)=E_{1}(F(t)) e^{i \omega(t)}, \frac{\partial u}{\partial x}\left(x_{0}, t\right)=E_{2}(F(t)) e^{i \omega(t)}, \text { for all } t \in W_{0}
$$

then

$$
I_{0} \subseteq I\left(x_{0} ; E_{1}(F(t)), E_{2}(F(t)) ; E_{3}(F(t))\right), \text { for all } t \in W_{0},
$$

and, for any $(x, t) \in I_{0} \times W_{0}$, we have

$$
u(x, t)=\sigma\left(x, x_{0} ; E_{1}(F(t)), E_{2}(F(t)) ; E_{3}(F(t))\right) e^{-i \omega_{0}(t) E_{3}(F(t))+i E_{4}(F(t))} .
$$

(b) Converse. Suppose that the relations (51) are true. Then: the function $u: I_{0} \times W_{0} \rightarrow \mathbb{C}$ defined, for any $(x, t) \in I_{0} \times W_{0}$, by the Formula (52) is a solution of the multitime NLSE (2), and satisfies the conditions (50).

Proof. For any $t \in W_{0}$, we have

$$
\begin{aligned}
& h^{\alpha}(t) \frac{\partial \omega}{\partial t^{\alpha}}(t)=-E_{3}(F(t)) h^{\alpha}(t) \frac{\partial \omega_{0}}{\partial t^{\alpha}}(t)- \\
& -\omega_{0}(t) h^{\alpha}(t) \frac{\partial}{\partial t^{\alpha}}\left(E_{3}(F(t))\right)+h^{\alpha}(t) \frac{\partial}{\partial t^{\alpha}}\left(E_{4}(F(t))\right) . \\
& \text { Hence } h^{\alpha}(t) \frac{\partial \omega}{\partial t^{\alpha}}(t)=-E_{3}(F(t)) .
\end{aligned}
$$

(a) We use Proposition 9.

For any $(x, t) \in I_{0} \times W_{0}$, the relation (37) is satisfied, and we deduce that $\varphi(x, t) \in I_{3}$. The relation (39) is now equivalent to

$$
E_{3}(F(t)) \cdot \varphi(x, t)+\frac{\partial^{2} \varphi}{\partial x^{2}}(x, t)+f\left(x,(\varphi(x, t))^{2}\right) \varphi(x, t)=0
$$

We remark that for all $t \in W_{0}$, the function $\varphi(\cdot, t): I_{0} \rightarrow I_{3}$ is a solution of the Equation (49), with parameter $k=E_{3}(F(t))$. Since $\varphi\left(x_{0}, t\right)=E_{1}(F(t))$ and $\frac{\partial \varphi}{\partial x}\left(x_{0}, t\right)=E_{2}(F(t))$, it follows that $I_{0} \subseteq I\left(x_{0} ; E_{1}(F(t)), E_{2}(F(t)) ; E_{3}(F(t))\right)$, and $\varphi(x, t)=\sigma\left(x, x_{0} ; E_{1}(F(t)), E_{2}(F(t)) ; E_{3}(F(t))\right)$, i.e., $u$ has the form in conclusion.

$(b)$ Let $\varphi(x, t):=\sigma\left(x, x_{0} ; E_{1}(F(t)), E_{2}(F(t)) ; E_{3}(F(t))\right), x \in I_{0}, t \in W_{0}$.

Since $\sigma\left(x, x_{0} ; E_{1}(F(t)), E_{2}(F(t)) ; E_{3}(F(t))\right) \in I_{3}$, it immediately follows that $(\varphi(x, t))^{2} \in I_{2}$, i.e., the relation (37).

The relation (38) is obviously fulfilled because for every $x$ fixed, $\varphi(x, \cdot)$ is a function that depends on $F(\cdot)$.

The relation (39) is equivalent to the relation (53), which is satisfied, because the function $\sigma\left(\cdot, x_{0} ; E_{1}(F(t)), E_{2}(F(t)) ; E_{3}(F(t))\right)$ is a solution of the Equation (49), with parameter $k=E_{3}(F(t))$.

The relations $\varphi\left(x_{0}, t\right)=E_{1}(F(t))$ and $\frac{\partial \varphi}{\partial x}\left(x_{0}, t\right)=E_{2}(F(t))$ are obvious, hence the initial conditions (50) are also true.

In the conditions of Theorem 1 , we denote $v(t ; k):=e^{-i k \omega_{0}(t)}$. The function $v(\cdot ; k)$ is a solution of the Equation (5); we have also $|v(t ; k)|=1$.

Hence the solution of Theorem 1 is written

$$
u(x, t)=\sigma\left(x, x_{0} ; E_{1}(F(t)), E_{2}(F(t)) ; E_{3}(F(t))\right) \cdot v\left(t ; E_{3}(F(t))\right) \cdot e^{i E_{4}(F(t))} .
$$


This is a solution similar to those in Remark 4 .

Proposition 12 and Theorem 1 say that, locally, the solutions of the multitime NLSE (2) whose argument does not depend on $x$, are only those described in Remark 4 (if we assume in addition that $\left.u\left(x_{0}, t_{0}\right) \neq 0\right)$.

Remark 8. For $m=1$, we easily find that the results obtained in this article remain true under the following conditions:

- the functions $\Psi$, respectively $\Psi_{j}$, which appear in Propositions 2-7, are taken constants;

- the functions $E_{j}$, which appear in Remark 4 , in Section 3, in Proposition 12, and in Theorem 1 are taken constant; the functions $F_{1}, F_{2}, \ldots, F_{m-1}$ are no longer considered in the case $m=1$ (instead of $E_{j}(F(t))$ is put the constant $E_{j}$ ).

\section{Multitime NLSE in Riemannian Setting}

From the physical point of view, it would be more important to further study a multitime NLSE in Riemannian setting, which is still an open problem. For that, let $(M, g)$ be a smooth compact Riemannian manifold of dimension $n$ (particularly, $n=2,3$ ) without boundary. In Riemannian setting, the multitime NLSE is

$$
i h^{\alpha}(x, t) \frac{\partial u}{\partial t^{\alpha}}+\Delta_{g} u+f\left(x,|u|^{2}\right) u=0, \quad(x, t) \in M \times \mathbb{R}^{m} .
$$

Let $d x_{g}$ denotes the volume element of the compact Riemannian manifold $(M, g)$ and

$$
|u|^{2}=u \bar{u},\left|\nabla_{g} u\right|_{g}^{2}=\left\langle\nabla_{g} u, \nabla_{g} \bar{u}\right\rangle_{g} .
$$

Open problem Find the minimum of the multitemporal energy functional

$$
E(t)=\frac{1}{2} \int_{M}\left(|u|^{2}+\left|\nabla_{g} u\right|_{g}^{2}\right) d x_{g},
$$

constrained by the multitime NLSE (54).

Hint To solve this problem we need techniques from variational calculus.

This multitime NLSE is mainly concerned with the interface between Riemannian geometry and quantum mechanics, but it leads, in a natural way, to questions of functional analysis related to the theory of operators on Hilbert spaces. In some respects these problems are similar to those studied in the standard Euclidean case, but depending on the Riemannian metric $g$ these might go beyond and provide new aspects to the problem.

\section{Conclusions}

The exact solutions of multitime NLSE (with oblique derivative) are closely related to the orbits of the direction vector field $h$. Our techniques for finding these solutions started from this important idea applied to PDEs that contain directional derivative. We follow a different route than those in the papers [1-19] and carry out the calculations to obtain significative exact solutions of multitime NLSE. This computational paper and the obtained results show that our methods are simple, efficient, straightforward and powerful. Moreover, the presented methods can be employed in many other types of nonlinear PDEs arising in mathematics, mathematical physics, engineering and economics.

The linearization of the multitime NLSE around a solution returns to the linearization of the function $f$. Linearization offers patterns of solutions that approximate the solutions of the original equation (this corresponds to studying the tangent space at a point of all solutions moduli space).

We hope that this paper will open a door for many readers to a multitime intriguing topic that is an active field of current research, especially in the Riemannian formulation of multitime nonlinear Schrödinger's PDE. 
Author Contributions: Conceptualization, C.G., C.U. and L.L.P.; methodology, all authors; writingoriginal draft preparation, C.G., C.U. and L.L.P.; writing-review and editing, C.G., C.U.; validation, C.U. All authors have read and agreed to the published version of the manuscript.

Funding: This research received no external funding.

Institutional Review Board Statement: Not applicable.

Informed Consent Statement: Not applicable.

Data Availability Statement: Not applicable.

Acknowledgments: We are grateful to Ionel Tevy for interesting proposals on improving our point of view on multitime NLSE. The authors would also like to thank Oana-Maria Pastae, "Constantin Brancusi" University of Tg-Jiu, for many useful and stimulating discussions regarding expressing the Romanian mathematical text in English.

Conflicts of Interest: The authors declare no conflict of interest.

\section{References}

1. Petrescu, L. Multitime Schrödinger spatial solitons. UPB Sci. Bull. Ser. A 2015, 77, 3-8

2. Genoud, F. An inhomogeneous, $L^{2}$ critical, nonlinear Schrödinger equation. Z. Anal. Ihre Anwendungen 2012, 31, 283-290 [CrossRef]

3. Genoud, F. Global bifurcation for asymptotically linear Schrödinger equations. Nonlinear Differ. Equations Appl. 2013, 20, 23-35 [CrossRef]

4. Genoud, F.; Stuart, C.A. Schrödinger equations with a spatially decaying nonlinearity: Existence and stability of standing waves. Discret. Contin. Dyn. Syst. Ser. A 2008, 21, 137-186 [CrossRef]

5. Genoud, F. Existence and orbital stability of standing waves for some nonlinear Schrödinger equations, perturbation of a model case. J. Differ. Equations 2009, 246, 1921-1943 [CrossRef]

6. Hodgson, M.J.P. Analytic Solution to the Time-Dependent Schrödinger Equation for the One-Dimensional Quantum Harmonic Oscillator with an Applied Uniform Field. Available online: https://www-users.york.ac.uk/ mjph501/Publications/MJP_Hodgson_analytic_ solution_2021.pdf (accessed on 29 June 2021).

7. Ishkhanyan, A.M. Exact solution of the Schrödinger equation for the inverse square root potential $V_{0} / \sqrt{x}$. Europhys. Lett. 2015, 112, 10006. [CrossRef]

8. Lienert, M.; Petrat, S.; Tumulka, R. Multi-Time Wave Functions, an Introduction; Springer: Berlin/Heidelberg, Germany, 2020

9. Matei, L.; Udriste, C. Construction of multitime Rayleigh solitons. UPB Sci. Bull. Ser. A 2014, 76, 29-43

10. Matei, L.; Udriste, C.; Ghiu, C. Multitime Boussinesq solitons. Int. J. Geom. Methods Mod. Phys. 2012, 9, 1250031 [CrossRef]

11. Petrat, S.; Tumulka, R. Multi-time equations, classical and quantum. Proc. R. Soc. A 2014, 470. [CrossRef] [PubMed]

12. Udriste, C.; Petrescu, L.; Matei, L. Multitime reaction-diffusion solitons. Balkan J. Geom. Appl. 2012, 17, 115-128

13. Riahi, N. Solving the time-dependent Schrödinger equation via Laplace transform. Quantum Stud. Math. Found. 2017, 4, 103-126. [CrossRef]

14. Yang, F.; Ning, Z.-H.; Chen, L. Exponential stability of the nonlinear Schrödinger equation with locally distributed damping on compact Riemannian manifold. Adv. Nonlinear Anal. 2021, 10, 569-583. [CrossRef]

15. Aksenov, A.V.; Polyanin, A.D. Methods for constructing complex solutions of nonlinear PDEs using simpler solutions. Mathematics 2021, 9, 345. [CrossRef]

16. Ghiu, C.; Tuliga, R.; Udriste, C.; Tevy, I. Discrete diagonal recurrences and discrete minimal submanifolds. Balkan J. Geom. Appl. 2015, 20, 49-64

17. Ghiu, C.; Tuliga, R.; Udriste, C.; Tevy, I. Floquet theory for multitime linear diagonal recurrence. UPB Sci. Bull. Ser. A 2016, 78, 3-8

18. Ghiu, C.; Tuliga, R.; Udriste, C. Discrete multiple recurrence. UPB Sci. Bull. Ser. A 2019, 81, 25-38

19. Ghiu, C.; Tuliga, R.; Udriste, C. Linear discrete multitime multiple recurrence. UPB Sci. Bull. Ser. A 2020, 82, 29-40 